\title{
Nanoscale
}

Check for updates

Cite this: Nanoscale, 2020, 12, 20002

Received 17th June 2020

Accepted 3rd September 2020

DOI: 10.1039/d0nr04592f

rsc.li/nanoscale

\section{A versatile UCST-type composite microsphere for image-guided chemoembolization and photothermal therapy against liver cancer $\dagger$}

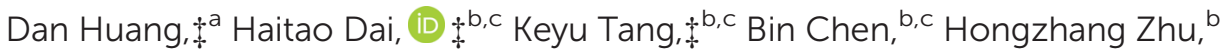 \\ Dubo Chen, ${ }^{d}$ Nan Li, ${ }^{b}$ Yingzhao Wang, ${ }^{e}$ Chuwei Liu, ${ }^{\text {e }}$ Yonghui Huang, ${ }^{b}$ \\ Jianyong Yang, ${ }^{b, c}$ Chao Zhang, (iD *a,c Run Lin (iD *b,c and Weiling He*c,e
}

\begin{abstract}
The development of novel chemoembolization agents to improve the treatment efficacy of transarterial chemoembolization (TACE) against liver cancer remains an urgent need in clinical practice. Herein, a versatile composite microsphere with upper critical solution temperature (UCST) properties was prepared to encapsulate polydopamine coated superparamagnetic iron oxide nanoparticles (SPIONAPDA) and doxorubicin for simultaneous chemoembolization and photothermal therapy. The microspheres were spherical with an average diameter of 100-300 $\mu \mathrm{m}$ and exhibited favorable drug loading capability as well as strong photothermal effect. Strikingly, synergistic enhancement of photothermal therapy and chemotherapy against chemoresistant liver cancer cells was achieved. The in vivo therapeutic efficacy and safety evaluations were performed using rabbit VX2 liver tumor models. It was revealed that a single treatment of the combination of TACE and photothermal procedure resulted in $87.5 \%$ complete response and $12.5 \%$ partial response for the microsphere group, whereas all tumors in the control group progressed rapidly. Contrast-enhanced computed tomography (CT) evaluation indicated that the tumor diameter decreased by $91.5 \%$ after treatment, while that in the control group increased by $86.5 \%$. The pathology-proven tumor necrotic rate was $87.2 \%$, which significantly surpassed that of $65.2 \%$ in the control group. Furthermore, serum liver enzyme and biochemical studies indicated a temporary liver injury which can be fully recovered. Our findings demonstrated that this microsphere may be advantageous for enhancing therapeutic efficacy of TACE against liver cancer.
\end{abstract}

riti/nanoscale

\section{Introduction}

Liver cancer, of which the major pathological type is hepatocellular carcinoma, remains a great challenge with dismal prognosis and constant increase in mortality and morbidity. ${ }^{1,2}$ The stealth onset of the liver cancer makes it difficult to diagnose

\footnotetext{
${ }^{a}$ School of Biomedical Engineering, Sun Yat-Sen University, Shenzhen, Guangdong, 518107, China

${ }^{b}$ Department of Radiology, The First Affiliated Hospital, Sun Yat-sen University, Guangzhou, Guangdong, 510080, China. E-mail: linrun5@mail.sysu.edu.cn ${ }^{c}$ Center for Precision Medicine, Sun Yat-Sen University, Guangzhou, Guangdong, 510080, China.E-mail:zhchao9@mail.sysu.edu.cn

${ }^{d}$ Department of Laboratory Medicine, The First Affiliated Hospital, Sun Yat-sen University, Guangzhou, Guangdong, 510080, China

${ }^{e}$ Department of Gastrointestinal Surgery, The First Affiliated Hospital, Sun Yat-sen University, Guangzhou, Guangdong, 510080, China.

E-mail: hewling@mail.sysu.edu.cn

$\dagger$ Electronic supplementary information (ESI) available. See DOI: 10.1039/ d0nr04592f

$\$$ These authors contributed equally to this work.
}

at an early stage when radical therapy is available, leaving non-curative treatments such as transarterial chemoembolization (TACE) the only option for these patients. ${ }^{3,4}$ TACE is the first-line treatment for unresectable intermediate-stage liver cancer as recommended by numerous guidelines, and studies are in progress to explore the feasibility of extending its application for liver cancer of other stages. ${ }^{4,5}$ TACE relies on chemoembolization agents, for example microspheres, to simultaneously deprive blood supply by blockage of tumor feeding vessels and deliver chemotherapeutic drugs, thereby inducing liver cancer cell necrosis and tumor regression., 6 Therefore, the performance of the chemoembolization vectors is the key determinant of TACE outcome. ${ }^{8-10}$

The major goal of TACE is to achieve complete tumor necrosis, thus providing patients with survival benefits as much as possible. It has been demonstrated that the magnitude of tumor necrosis, in terms of treatment response which is usually assessed by contrast enhanced imaging studies, correlated closely with prognosis. ${ }^{11-15}$ Unfortunately, TACE alone can hardly eliminate tumor cells entirely, as only around half 
of the patients who were subjected to TACE achieved complete response (CR) according to the imaging study. ${ }^{11}$ Pathological complete necrosis is even more difficult to achieve as viable tumor cells can be detected in the majority of the lesions after TACE treatment. ${ }^{12-15}$ The residual tumor cells account for the rapid tumor recurrence and progression that substantially deteriorate the prognosis. However, little improvement has been made that chemoembolization agents of new generation such as drug eluting beads (DEBs) failed in providing better overall survival (OS) for patients compared to conventional agents. ${ }^{5}$ Therefore, the development of novel vehicles to enhance tumor eradication remains an urgent need for improving the treatment efficacy of liver cancer.

To improve tumor elimination, treatment strategies have to abrogate the biological behaviors that contribute to the survival advantages of liver cancer cells, in particular chemoresistance. DEB release per loaded drugs via ion-exchange mechanism in a slow and sustained release pattern to deliver chemotherapeutic agents toward tumor lesions continuously. ${ }^{8-10}$ However, being intrinsically drug-resistant in nature, liver cancer is notorious for being refractory to either systemic or transarterial infusion chemotherapy. ${ }^{4,16}$ Furthermore, conclusive evidence supporting the superiority of TACE over transarterial embolization (TAE) is still absent, suggesting the poor performance of chemotherapy for liver cancer. ${ }^{17,18}$ Chemoresistance remains a vital obstruction for both systemic and locoregional cytotoxic treatment to take effect. Among strategies to overcome the chemo-refractory, incorporation of thermotherapy as an additional treatment modality shows great potential for fulfilling the pressing need. Thermotherapy per se exerts a robust tumor eradicative effect that a slight increase in temperature is adequate to destroy tumor cells without causing severe adverse effects. ${ }^{19}$ Furthermore, hyperthermia has a potent chemo-sensitizing effect, a feature that is of particular importance for liver cancer therapy. ${ }^{19}$ On the other hand, utilization of stimuli-responsive polymers to fabricate drug delivery platforms is a proven approach to achieve a controllable release pattern and ultimately more favorable treatment outcomes. ${ }^{20,21}$ Specifically, polymers with upper critical solution temperature (UCST) properties are perfectly suitable for the construction of the above-mentioned photothermal microsphere. The solubility as well as the drug release rate of the UCST polymer increase substantially when the temperature exceeds a certain threshold that facilitates hyperthermia and chemotherapy to function simultaneously, which may interact synergistically to produce a remarkable enhancement. $^{22-25}$

In this work, a novel doxorubicin loaded versatile composite microsphere with UCST properties and encapsulated polydopamine coated superparamagnetic iron oxide nanoparticles (SPION@PDA) was prepared for simultaneous chemoembolization and photothermal therapy. Comprehensive characterization studies were performed to fully investigate the physicochemical and mechanical properties of the prepared microsphere. In vitro photothermal effect, photothermal stability, and drug loading and releasing profiles were investigated.
Biocompatibility was assessed by cytotoxicity and hemolysis evaluations. The cellular anti-tumor effect was explored on HepG2 human liver cancer cells and chemoresistant HepG2/ ADR cells. Rabbit VX2 liver tumor models were established and TACE procedures were implemented using the developed poly(acrylamide-co-acrylonitrile)/(SPION@PDA) (P(AAm-co-AN)/ (SPION@PDA)) microspheres. In vivo photothermal and magnetic resonance imaging (MRI) contrast effects of the microspheres were also determined. Laser irradiation was given right after the TACE procedures for the treatment group ( $n=$ $8)$. The therapeutic efficacy was assessed at one $(n=4)$ and two $(n=4)$ weeks after treatment by dual-phase dynamic contrastenhanced computed tomography (CT) scanning according to modified Response Evaluation Criteria in Solid Tumors (mRECIST) $^{26}$ and was further confirmed by pathological examinations. Three rabbits with VX2 liver tumor were employed as the control. The therapeutic safety was determined by liver enzyme and biochemical tests.

\section{Experimental section}

\section{Materials}

Analytical grade (A.R.) acrylamide (AAm), acrylonitrile (AN), 2,2'-azoisobutyronitrile (AIBN), and $N, N^{\prime}$-methylene bisacrylamide (MBAAm) were purchased from Tianjin Damao Chemical Reagent Factory (Tianjin, China) and were re-crystallized in ethanol or acetone before use. Dopamine hydrochloride (DA, Sigma-Aldrich, USA), 3-(4,5-dimethyl-thiazol-2-yl)-2,5-diphenyl tetrazolium bromide (MTT, Sigma-Aldrich, USA), dimethyl sulfoxide (DMSO, A.R., Sinopharm Chemical Reagent Co., Ltd, Shanghai, China), superparamagnetic iron oxide nanoparticles (SPION, 99.9\%, Beijing Deke Daojin Science and Technology Co., Ltd, Beijing, China), doxorubicin hydrochloride (DOX, Macklin, Shanghai, China), mineral oil (Adamas, Shanghai, China), emulsifier EM90 (Adamas, Shanghai, China), and pentobarbital sodium (>99\%, MSD China, Shanghai, China) were used as received. Human hepatocellular carcinoma cells (BEL-7402 and HepG2) and human normal hepatocytes (L02) were obtained from Shanghai Cell Bank of the Chinese Academy of Sciences (Shanghai, China). Doxorubicin-resistant HepG2/ADR human hepatocellular carcinoma cells were purchased from Shanghai SUER Biological Technology Co., Ltd (Shanghai, China). VX2 tumor tissues, an anaplastic squamous cell carcinoma which was originally derived from a virusinduced papilloma in rabbits, were obtained from Guangzhou Jennio Biotech Co., Ltd (Guangzhou, China). High-glucose Dulbecco's modified Eagle's medium (DMEM, Hyclone, USA), Roswell Park Memorial Institute 1640 (RPMI-1640) medium, fetal bovine serum (FBS, Gibco, USA) and penicillin-streptomycin (Pen/Strep, Tianjin Haoyang Biological Manufacture Co., Ltd, China) were used for cell culture.

\section{Preparation of microspheres}

The UCST-type composite microspheres were fabricated by inverse emulsion copolymerization of AAm, AN, and MBAAm, 


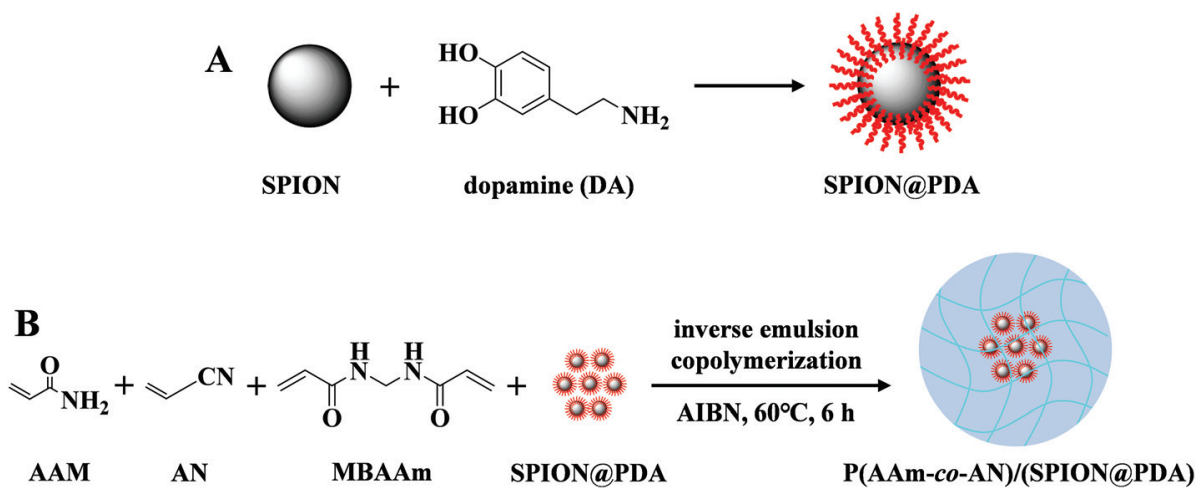

Fig. 1 Preparation route of (A) SPION@PDA nanoparticles and (B) P(AAm-co-AN)/(SPION@PDA) microspheres.

in the presence of SPION@PDA (Fig. 1). Detailed preparation and characterization of SPION@PDA are available in the ESI. $\dagger$ To prepare the microspheres, $50 \mu \mathrm{L}$ of $\mathrm{AN}$ and $10 \mathrm{mg}$ of SPION@PDA were added to $0.5 \mathrm{~mL}$ solution of $100 \mathrm{mg}$ of AAm in deionized water and the mixture was stirred for one hour at room temperature. Afterward, $5 \mathrm{mg}$ of MBAAm and $1 \mathrm{mg}$ of AIBN were added and stirred for additional 30 minutes. Subsequently, the mixture was added dropwise into $1.2 \mathrm{~mL}$ of mineral oil with $2 \%$ of EM90 as an emulsifier, stirring at 400 rpm for 6 hours at $60{ }^{\circ} \mathrm{C}$. The product was separated by centrifugation and washed sequentially with petroleum ether, ethanol, and deionized water three times. Finally, the obtained $\mathrm{P}(\mathrm{AAm}-\mathrm{co}-\mathrm{AN}) /(\mathrm{SPION} @ \mathrm{PDA})$ microspheres were lyophilized for storage. Blank $\mathrm{P}(\mathrm{AAm}-\mathrm{co}-\mathrm{AN})$ microspheres were prepared following similar procedures of control experiments.

\section{Characterization studies}

An optical microscope (DM2500p, Leica, Germany) was used to observe the size and morphology of the swelling microspheres, and a scanning electron microscope (SEM, JSM-6330F, JEOL, Tokyo, Japan) was used to observe the size and morphology of the lyophilized microspheres. The infrared spectra of the microspheres and other substances were obtained using a Nicolet 6700 Fourier transform infrared (FTIR) spectrophotometer (Thermo Scientific, USA). Elemental microanalysis of the surface of microspheres was performed by SEM with a Quanta 400 FEG energy dispersive X-ray spectrometer (SEM-EDS, FEI/OXFORD/HKL, France). The content of SPION@PDA encapsulated in the microspheres was quantified using an SMP/PF7548/MET thermogravimetric analyzer (TGA, METTLER TOLEDO, USA) from ambient temperature to $900{ }^{\circ} \mathrm{C}$ with a heating rate of $15{ }^{\circ} \mathrm{C}$ min $^{-1}$ under an $\mathrm{O}_{2}$ flow of $50 \mathrm{~mL}$ $\mathrm{min}^{-1}$. A dimension icon atomic force microscopy (AFM, ICON, Bruker, USA) assisted nanoindentation on a vibration isolation table was used to measure the Young's modulus of the microspheres from $25^{\circ} \mathrm{C}$ to $55^{\circ} \mathrm{C}$ on a heating platform. The spring constant $k$ of the non-conductive silicon nitride cantilever (MLCT-A, Bruker, USA) was $0.1167 \mathrm{~N} \mathrm{~m}^{-1}$ and the probe velocity was $2 \mu \mathrm{m} \mathrm{s}^{-1}$, attached with a polystyrene bead $(5.0 \mu \mathrm{m}$ in diameter). At least five samples were chosen for the test, and each microsphere was scanned thrice at each tested temperature under an optical microscope which was used to position the AFM tip. ${ }^{27}$ The Young's modulus was derived using the NanoScope Analysis software (Bruker, USA). The UCST value of the P(AAm-Co-AN) microsphere was determined by differential scanning calorimetry (DSC, DSC 250, TA instruments, Waters, LLC, USA) under a $\mathrm{N}_{2}$ atmosphere from $30^{\circ} \mathrm{C}$ to $90{ }^{\circ} \mathrm{C}$ with a heating rate of $5{ }^{\circ} \mathrm{C} \mathrm{min}^{-1}$. To verify the MRI contrast effect of the $\mathrm{P}(\mathrm{AAm}-\mathrm{co}-\mathrm{AN}) /(\mathrm{SPION} @ \mathrm{PDA})$ in vitro, samples with a series of gradient concentrations $(0,0.625$, $1.25,2.50,5.00,10.0,20.0$, and $40.0 \mathrm{mg} \mathrm{mL}^{-1}$ ) were prepared by dispersing the microspheres into $1.5 \%$ agarose solution in $1.5 \mathrm{~mL}$ vials. The samples were well-mixed at $4{ }^{\circ} \mathrm{C}$ to a solidified state and were subjected to MRI scanning using a 3 Tesla whole body system (Magnetom Verio, Siemens Healthcare Sector, Erlangen, Germany) and a 32 phased-array head coil. Transversal $T_{2}$-weighted fast spin echo (FSE) images were obtained using the following parameters: TR, $3600 \mathrm{~ms}$; TE, $87 \mathrm{~ms}$; slice thickness, $1.0 \mathrm{~mm}$; flip angle, $180^{\circ}$; field of view (FOV), $100 \mathrm{~mm} \times 60 \mathrm{~mm}$. The images were analyzed using Radiant DICOM viewer 5.0.1 (Medixant, Poznan, Poland). The changes in diameter of the $\mathrm{P}(\mathrm{AAm}-\mathrm{co}-\mathrm{AN})$ microspheres during the heating-cooling procedure were observed and measured in order to confirm the UCST properties. Additional characterization data are available in the ESI. $\dagger$

\section{In vitro photothermal effect and photothermal stability}

The photothermal effect of the nanoparticles and microspheres was evaluated using near infrared (NIR) laser irradiation (Beijing Viasho Technology Co., Ltd, China) at $808 \mathrm{~nm}$. For the SPION and SPION@PDA nanoparticles, $1.0 \mathrm{~mL}$ of the suspensions were irradiated at a power density of $1.0 \mathrm{~W} \mathrm{~cm} \mathrm{~cm}^{-2}$ for 10 minutes at a concentration of $3.0 \mathrm{mg}$ $\mathrm{mL}^{-1}$. For P(AAm-co-AN)/(SPION@PDA) microspheres, suspensions with a volume of $1.0 \mathrm{~mL}$ were irradiated under stirring at varied power levels $\left(0.5,1.0\right.$, and $\left.1.5 \mathrm{~W} \mathrm{~cm}^{-2}\right)$ for 10 minutes with a microsphere concentration of $40 \mathrm{mg} \mathrm{mL}$, or at different concentrations $\left(1,10,20\right.$, and $\left.40 \mathrm{mg} \mathrm{mL}^{-1}\right)$ with a set power of $1.0 \mathrm{~W} \mathrm{~cm}^{-2}$. The temperatures were measured using an HT-9815 thermocouple thermometer (Dongguan Xintai 
Instrument Co., Ltd, Dongguan, China) which was inserted into the suspension. $\mathrm{P}(\mathrm{AAm}-\mathrm{co}-\mathrm{AN})$ microsphere suspension and saline were used as the control. Repeated irradiationcooling cycles were carried out to evaluate the photostability of the $\mathrm{P}(\mathrm{AAm}-\mathrm{co}-\mathrm{AN}) /(\mathrm{SPION} @ \mathrm{PDA})$ microspheres. Briefly, the microsphere suspension was irradiated for 5 minutes at a power density of $1.0 \mathrm{~W} \mathrm{~cm}^{-2}$ and was then naturally cooled to room temperature (around $25{ }^{\circ} \mathrm{C}$ ) before the next irradiation. The irradiation-cooling cycle was performed 5 times.

\section{Drug loading and release profiles}

Doxorubicin, a broad-spectrum chemotherapy drug, was selected for investigating the drug loading and release profiles of the microspheres. Briefly, $20 \mathrm{mg}$ of lyophilized microspheres were dispersed in $0.5 \mathrm{~mL}$ of DOX solution $(4.0 \mathrm{mg}$ $\mathrm{mL}^{-1}$ in deionized water) at $50{ }^{\circ} \mathrm{C}$, and the suspension was shaken at $100 \mathrm{rpm}$ for 24 hours. Then, the microspheres were collected by repeated centrifugations and were washed with saline four times. The supernatant and the washing saline were transferred to a $25 \mathrm{~mL}$ volumetric flask. The concentration and the amount of DOX in the volumetric flask were determined using a spectrophotometer with a working wavelength of $482 \mathrm{~nm}$. The amount of DOX loaded in the microspheres was derived by the deduction of the amount of DOX in the volumetric flask from the total amount of DOX. Four samples were tested for each group. The drug-loading efficiency (LE) and encapsulation efficiency (EE) were defined using the following equations (eqn (1) and (2)):

$$
\begin{aligned}
& \mathrm{LE}(\%)=\frac{\text { mass of the encapusulated DOX }}{\text { total mass of the microspheres }} \times 100 \\
& \mathrm{EE}(\%)=\frac{\text { mass of the encapusulated DOX }}{\text { mass of the initial DOX }} \times 100
\end{aligned}
$$

To investigate the release behavior of the microspheres, $20 \mathrm{mg}$ of DOX loaded microspheres were dispersed in $2.0 \mathrm{~mL}$ of saline solution in a $10 \mathrm{~mL}$ centrifuge tube and the suspension was shaken at $100 \mathrm{rpm}$ and $37^{\circ} \mathrm{C}$ for 24 hours. To determine the concentration of DOX in the release medium, $0.2 \mathrm{~mL}$ of the supernatant was sampled and replaced with an equal volume of fresh saline. The concentration and amount of DOX in the supernatant were measured using a spectrophotometer. The accumulative release rate of DOX from microspheres was calculated according to the following equation (eqn (3)):

$$
\begin{aligned}
& \text { Accumulative DOX release }(\%) \\
& =\frac{\text { mass of the released DOX }}{\text { mass of the encapusulated DOX }} \times 100
\end{aligned}
$$

Photothermal triggered release of DOX from the microspheres was verified under repeated NIR laser irradiation ( $808 \mathrm{~nm}, 1.0 \mathrm{~W} \mathrm{~cm}^{-2}$, and 5 minutes). The DOX loaded microspheres were dispersed in $2.0 \mathrm{~mL}$ of saline in a $10 \mathrm{~mL}$ centrifuge tube. $0.2 \mathrm{~mL}$ of the supernatant was sampled and replaced with an equal volume of fresh saline at $0 \mathrm{~min}$, $60 \mathrm{~min}, 120 \mathrm{~min}$ (Laser “ON"), $125 \mathrm{~min}$ (Laser “OFF"), $180 \mathrm{~min}, 240 \mathrm{~min}$ (Laser “ON”), $245 \mathrm{~min}$ (Laser “OFF”),
$300 \mathrm{~min}, 360 \mathrm{~min}$ (Laser "ON"), 365 min (Laser "OFF"), and $420 \mathrm{~min}$. The supernatants were analyzed using a spectrophotometer at $482 \mathrm{~nm}$ to determine the concentrations of DOX and the accumulative release amount of DOX was derived according to the aforementioned equation (eqn (3)).

\section{Biocompatibility}

BEL-7402 and L02 cells were kept in RPMI1640, and HepG2 and HepG2/ADR cells were kept in DMEM. The cells were grown at $37{ }^{\circ} \mathrm{C}$ under a humidified atmosphere of $95 \%$ air and $5 \% \mathrm{CO}_{2}$. The complete culture medium was supplemented with $1 \%$ penicillin-streptomycin (Pen/Strep) and $10 \%$ fetal bovine serum, and specifically, the culture medium for HepG2/ADR cells contained $2.0 \mu \mathrm{g} \mathrm{mL} \mathrm{mL}^{-1}$ of DOX. Biocompatibility of the microspheres was determined by cytotoxicity and hemolysis assays. The cytotoxicity was evaluated by co-culture of BEL-7402, L02 and HepG2 cells with the extract of the microspheres and subsequent MTT assay. To prepare the extract, the microspheres were sterilized in $75 \%$ ethanol for 24 hours, rinsed with sterile phosphate buffered saline (PBS, with 1\% of Pen/Strep) three times every four hours, and lyophilized thereafter. The extract was prepared according to the ISO10993 criterion that the lyophilized microspheres were dispersed in the growth medium at a concentration of $200 \mathrm{mg} \mathrm{mL}{ }^{-1}$ for 24 hours, ${ }^{28}$ and the supernatant was collected for the cell culture. The cells were seeded into 96-well plates (5000 cells per well for BEL-7402, L02, and HepG2, and 10000 cells per well for HepG2/ADR, respectively) and incubated for 24 hours. Then, the culture medium was replaced by $100 \mu \mathrm{L}$ of the extract and the cells were incubated for another 24 hours. Subsequently, the extract of the microspheres was removed carefully and the cells were gently washed with PBS three times. Afterward, $100 \mu \mathrm{L}$ of fresh growth medium with $20 \mu \mathrm{L}$ of MTT $(5 \mathrm{mg}$ $\mathrm{mL}^{-1}$ ) was added into each well and incubated for 3 hours. The medium was removed and the cells were washed with PBS carefully before adding $150 \mu \mathrm{L}$ of DMSO into each well to dissolve formazan. The absorbance at $570 \mathrm{~nm}$ was read using a Synergy 4 microplate reader (BioTek, USA). The growth medium was used as the control. Each sample was set with 5 parallels, and the results were presented as mean \pm standard deviation.

Fresh whole blood was obtained from healthy donors in the First Affiliated Hospital, Sun Yat-sen University, for evaluating the hemolytic properties of the microspheres. In brief, $2.0 \mathrm{~mL}$ of whole blood was centrifuged at $5000 \mathrm{rpm}$ for $10 \mathrm{~min}$. The collected erythrocytes were rinsed with PBS three times and diluted in $10 \mathrm{~mL}$ of PBS. The microspheres were dispersed into the erythrocyte suspensions at concentrations of $3.125,6.25,12.5,25,50,100$, and $200 \mathrm{mg} \mathrm{mL}^{-1}$, respectively. The mixtures were incubated at $37{ }^{\circ} \mathrm{C}$ for 3 hours and were then centrifuged. The supernatants were transferred to a 96-well plate and subjected to the measurement of absorbance at $540 \mathrm{~nm}$ using a microplate reader. PBS and deionized water were employed as the negative $\left(A^{-}\right)$ and positive $\left(A^{+}\right)$control, respectively. The degrees of hemo- 
lysis $(H \%)$ were calculated using the following equation (eqn (4)):

$$
H(\%)=\frac{A_{\mathrm{M}}-A^{-}}{A^{+}-A^{-}}
$$

where $A_{\mathrm{M}}, A^{-}$, and $A^{+}$are the absorbances of the microsphere suspension, negative control, and positive control, respectively. Triplicate samples were tested for each data point, and the results were presented as mean \pm standard deviation.

\section{In vitro anti-tumor assay}

The in vitro anti-tumor performance of the microspheres, especially against chemoresistant tumor cells, was evaluated by MTT assay using HepG2 and HepG2/ADR cells. Complete growth medium with 1.0 or $2.0 \mu \mathrm{g} \mathrm{mL} \mathrm{mL}^{-1}$ of DOX was prepared, and the concentrations of the DOX loaded microspheres in the medium were adjusted accordingly to achieve the same initial DOX concentration. Seven groups were set to evaluate the anti-tumor performance, namely, blank control, laser irradiation only, $\mathrm{P}(\mathrm{AAm}-\mathrm{co}-\mathrm{AN}) /(\mathrm{SPION} @ \mathrm{PDA})$ microsphere, free DOX, DOX loaded P(AAm-co-AN)/(SPION@PDA) microsphere, $\mathrm{P}(\mathrm{AAm}-\mathrm{co}-\mathrm{AN}) /(\mathrm{SPION} @ \mathrm{PDA})$ microsphere with laser irradiation, and DOX loaded P(AAm-co-AN)/(SPION@PDA) microsphere with laser irradiation, which were abbreviated as Control, Laser, MS, DOX, MS-DOX, MS + Laser, and MS-DOX + Laser, respectively. Before tests, 5000 HepG2 cells or 10000 HepG2/ADR cells per well in $100 \mu \mathrm{L}$ of growth medium were seeded into a 96-well plate and incubated for 24 hours. The culture medium was replaced by an equal volume of the DOX or microsphere containing growth medium. Laser irradiation (wavelength of $808 \mathrm{~nm}$, power density of $1.0 \mathrm{~W} \mathrm{~cm}^{-2}$, and duration of 10 minutes) was given subsequently in the designated groups. The cells were incubated for another 24 hours, and the viabilities of the cells were determined by MTT assay.

\section{Rabbit VX2 liver tumor models}

All animal experimental protocols were reviewed and approved by the Animal Care Committee of Sun Yat-sen University (SYSU-IACUC-2019-000331) and the experiments were performed in accordance with the National Institutes of Health's guidelines for the use of experimental animals. Male New Zealand White rabbits weighing between 2.5 and $3.0 \mathrm{~kg}$ were obtained from Guangdong Medical Laboratory Animal Center (Guangzhou, China). In this study, the rabbits were anesthetized by intravenous injection of pentobarbital sodium $(3 \%$, $30 \mathrm{mg} \mathrm{kg}{ }^{-1}$ ) via the auricular vein and additional doses were given when necessary. All the surgical procedures were performed under sterile conditions, and $100 \mathrm{mg}$ of cefuroxime sodium (Zinacef $\AA$, GlaxoSmithKline, Verona, Italy) was injected intravenously after each procedure to prevent infection.

VX2 tumor tissue was defrosted upon receiving, minced into 1-2 $\mathrm{mm}$ fragments, and implanted into the hind limb muscle of carrier rabbits. The carrier rabbits were sacrificed by excessive anesthesia three weeks later. The tumors were excised, minced into 1-2 $\mathrm{mm}$ pieces, and placed in ice-cooled saline until implantation. The VX2 tumor strain was maintained by successive transplantation of tumor tissue into the hind limb of carrier rabbits.

To establish the liver tumor model, a mini-laparotomy was performed in the subxiphoid area of the recipient rabbit to expose the liver. An incision with a depth of approximately $10 \mathrm{~mm}$ was made in the left hepatic lobe of the recipient rabbit using an 18 Gauge needle. Several pieces of tumor tissue were implanted into the incision and subsequently a piece of gelatin sponge was placed on the wound to prevent tumor dislocation. Two weeks after tumor transplantation, dual-phase dynamic contrast-enhanced CT study was performed weekly to monitor the tumor growth using a 320-slice CT scanner (Aquilion ${ }^{\mathrm{TM}}$ ViSION, Canon Medical Systems, Otawara, Japan) and additional scanning was performed if necessary. The parameters of the scanning protocol were as follows: tube voltage, $80 \mathrm{kV}$; tube current, $40 \mathrm{~mA}$; slice thinness, $0.5 \mathrm{~mm}$; pixel spacing, $1.0 \mathrm{~mm}$; rotation time, $0.358 \mathrm{~s}$; FOV, $160 \mathrm{~mm}$. The contrast agent (iopromide, Ultravist ${ }^{\circledR} 370$, Bayer HealthCare Co. Ltd, Shanghai, China) was injected through the auricular vein $\left(0.5 \mathrm{~mL} \mathrm{~s}{ }^{-1}, 1.5 \mathrm{~mL} \mathrm{~kg}^{-1}\right)$. The images were processed with Radiant DICOM viewer 5.0.1. A total of 14 rabbits with appropriate tumor diameters and typical imaging features were prepared for in vivo experiments. Among them, one rabbit was employed for an in vivo MRI contrast effect study and two rabbits for in vivo photothermal evaluation. The remaining 11 rabbits were randomly classified into the treatment group $(n=8)$ and control group $(n=3)$, and the corresponding average tumor diameters were $15.03 \pm$ $3.4 \mathrm{~mm}$ and $16.03 \pm 0.61 \mathrm{~mm}$, respectively $(p>0.05)$.

\section{In vivo experiments}

The right femoral artery was surgically exposed and a 4-F sheath (Introducer II Radifocus, Terumo Corporation, Tokyo, Japan) was inserted into the femoral artery. The TACE procedures were performed under fluoroscopy guidance (Allura Xper FD20, Philips Medical Systems, Eindhoven, the Netherlands), using a 1.8-F microcatheter (NC-F863A, Terumo Corporation, Tokyo, Japan) and a 0.014-inch guide wire (PT Graphix $^{\mathrm{TM}}$, Boston Scientific, USA). Angiographies of the celiac artery, hepatic lobar and hepatic segmental artery were performed to clarify the vascular anatomy and to identify the feeding artery of the tumor. The catheter was then advanced super-selectively into the left hepatic artery and was placed into an appropriate position for subsequent TACE procedures (Fig. 5A, B and S2 $\dagger$ ). The suspension of DOX loaded P(AAm-coAN)/(SPION@PDA) was mixed with iopamidol-370 $(370 \mathrm{mg}$ iodine per $\mathrm{mL}$, Isovue $\AA 370$, Bracco, Milan, Italy) at a volume ratio of $1: 3$ to prepare a mixture with a microsphere concentration of $1.0 \mathrm{mg} \mathrm{mL}^{-1}$. The mixture was then carefully injected through the catheter under fluoroscopic monitor until disappearance of the tumor vessel staining. Repeated angiography confirmed the entire deprivation of the tumor blood supply in 7 of 8 rabbits in treatment groups (Fig. 5 and $\mathrm{S} 2 \dagger$ ). In order to avoid nontarget embolization and severe damage of the gallbladder, complete embolization was not achieved in 
one tumor due to the presence of collateral circulations between the left hepatic artery and the right hepatic arterygallbladder artery, which cannot be bypassed by catheter operation (Fig. S2A-C $\dagger$ ). This led to a suboptimal treatment outcome as discussed later. After completion of the injection, the sheath and catheter were removed, the common femoral artery was ligated, and the wound was sutured.

MRI scanning was performed in one rabbit one day before and three hours after TACE to evaluate the in vivo MRI contrast effect of P(AAm-co-AN)/(SPION@PDA) microspheres. Transversal $T_{2}$-weighted FSE images were obtained using the identical MRI scanning equipment for an in vitro study and an 8 phased-array head coil with the following parameters: TR, $4800 \mathrm{~ms}$; TE, $98 \mathrm{~ms}$; slice thickness, $1.0 \mathrm{~mm}$; flip angle, $150^{\circ}$; FOV, $220 \mathrm{~mm} \times 160 \mathrm{~mm}$. The images were analyzed using a Radiant DICOM viewer and ImageJ (National Institutes of Health, Bethesda, MD, USA). The average MRI signal intensity of the tumor was calculated from three tumor-containing slices.

Laparotomy was performed as soon as TACE was conducted to expose the left hepatic lobe with tumor. Two rabbits were employed to evaluate the in vivo photothermal effect of the $\mathrm{P}(\mathrm{AAm}-\mathrm{co}-\mathrm{AN}) /(\mathrm{SPION} @ \mathrm{PDA})$ microspheres. Laser irradiation with a wavelength of $808 \mathrm{~nm}$ and a power level of $1.0 \mathrm{~W} \mathrm{~cm}^{-2}$ was applied to the tumor for 10 minutes using a K808F11CD-4.000w semiconductor laser source (BWT Beijing Co., Ltd, Beijing China). Temperatures of the tumor surface and core regions during laser irradiation were measured using a Ti27 infrared thermal camera (Fluke, USA) and an HT-9815 thermocouple thermometer (XINTEST, Dongguan Xintai Instrument Co., Ltd, Dongguan, China), respectively. CT scanning was performed to ensure the appropriate location of the thermometer tip. For the 8 rabbits in the treatment group, laser irradiation $\left(808 \mathrm{~nm}, 1.0 \mathrm{~W} \mathrm{~cm} \mathrm{~cm}^{-2}\right.$, and 10 minutes) was applied on each side of the tumor and the temperatures were measured using an infrared thermal camera. Abdominal wound was sutured after treatment and the animals were monitored until fully recovered from anesthesia.

\section{Therapeutic efficacy and safety}

The therapeutic efficacy was evaluated by imaging and pathological studies. Dual-phase dynamic contrast-enhanced CT scans were carried out one week $(n=4)$ or two weeks $(n=4)$ after therapy for the treatment group, and two weeks $(n=3)$ after assignment for the control group, using the identical equipment and protocol. The therapeutic efficacy was determined according to modified Response Evaluation Criteria in Solid Tumors (mRECIST). ${ }^{26}$ All rabbits were sacrificed by excessive anesthesia after CT scanning and the liver segments with tumors were harvested. The samples were cut into half along the longest axis of the tumors and were fixed with $10 \%$ paraformaldehyde. Hematoxylin-eosin (HE) stanning was performed and the slides were scanned. The viable and the necrotic areas of the tumors were delineated using Caseviewer 3.0 (3DHistech Ltd, Budapest, Hungary) and the tumor necrotic ratio was calculated via the following equation (eqn (5)).

$$
N(\%)=\frac{N_{1}}{N_{2}} \times 100 \%
$$

where $N, N_{1}$, and $N_{2}$ are the tumor necrotic ratio, the area of necrotic tumor, and the area of tumor, respectively.

The therapeutic safety was evaluated by liver enzyme and biochemical tests including alanine aminotransferase (ALT, U $\mathrm{L}^{-1}$ ), aspartate aminotransferase (AST, $\mathrm{U} \mathrm{L} \mathrm{L}^{-1}$ ), alkaline phosphatase (ALP, $\mathrm{U} \mathrm{L}^{-1}$ ), gamma glutamyl transpeptidase (GGT, U $\mathrm{L}^{-1}$ ), albumin (ALB, $\mathrm{g} \mathrm{L}^{-1}$ ), and serum total bilirubin (TBIL, $\left.\mu \mathrm{mol} \mathrm{L} \mathrm{L}^{-1}\right)$. Blood samples were collected right before treatment, and 1, 4, 7, and 14 days post treatment, respectively.

\section{Statistical analysis}

Statistical analysis was performed using SPSS 22.0 software (SPSS Inc., Chicago, USA). Data were presented as mean value \pm standard deviation, and comparison was determined by the $t$-test. $P<0.05$ was considered statistically significant.

\section{Results and discussion}

\section{Characterization studies}

The SPION@PDA nanoparticles, P(AAm-co-AN) microspheres, and P(AAm-co-AN)/(SPION@PDA) microspheres were successfully prepared as demonstrated by the following characterization studies. The SPION@PDA nanoparticles were characterized by X-ray photoelectron spectroscopy (XPS), TGA, FTIR, SEM, and transmission electron microscopy (TEM) (Fig. 2 and $\mathrm{S} 1 \dagger)$. In the XPS spectra, the additional peak at $400.08 \mathrm{eV}$ represented the presence of nitrogen, indicating the successful coating of PDA (Fig. 2A and S1A $\dagger$ ), which were concordant with the TEM analysis (Fig. S1C $\dagger$ ). The temperatures of SPION@PDA and SPION solutions increased from $26{ }^{\circ} \mathrm{C}$ to $49^{\circ} \mathrm{C}$ and $43^{\circ} \mathrm{C}$, respectively, confirming the enhanced photothermal effect by PDA (Fig. 2B). SEM and TEM were employed to observe the morphology and size distribution of the SPION@PDA nanoparticles. It was shown that the average diameter of SPION@PDA was approximately 100 nm (Fig. S1B and $\mathrm{S} 1 \mathrm{C} \dagger)$.

The optical micrographs revealed a spherical appearance and smooth surface of the swelling $\mathrm{P}(\mathrm{AAm}-\mathrm{co}-\mathrm{AN}) /$ (SPION@PDA) and P(AAm-co-AN) microspheres in the size range of 100-300 $\mu \mathrm{m}$ (Fig. $2 \mathrm{C}$ and $\mathrm{S} 1 \mathrm{D} \dagger$ ). Embedded SPION@PDA was viewed inside the $\mathrm{P}(\mathrm{AAm}-\mathrm{co}-\mathrm{AN}) /$ (SPION@PDA) microspheres but not in P(AAm-co-AN) microspheres in optical micrographs (Fig. 2C and S1D †). The SEM micrographs revealed that the size of the lyophilized microspheres ranged between 10 and $30 \mu \mathrm{m}$ with a smooth spherical appearance (Fig. 2D and $\mathrm{S} 1 \mathrm{E}^{\dagger}$ ).

In the FTIR spectrum of SPION@PDA, the strong vibrational absorption band at $580 \mathrm{~cm}^{-1}$ (Fe-O vibration mode) was gradually weakened compared to that of SPION, indicating the successful coating of PDA. The peaks at 

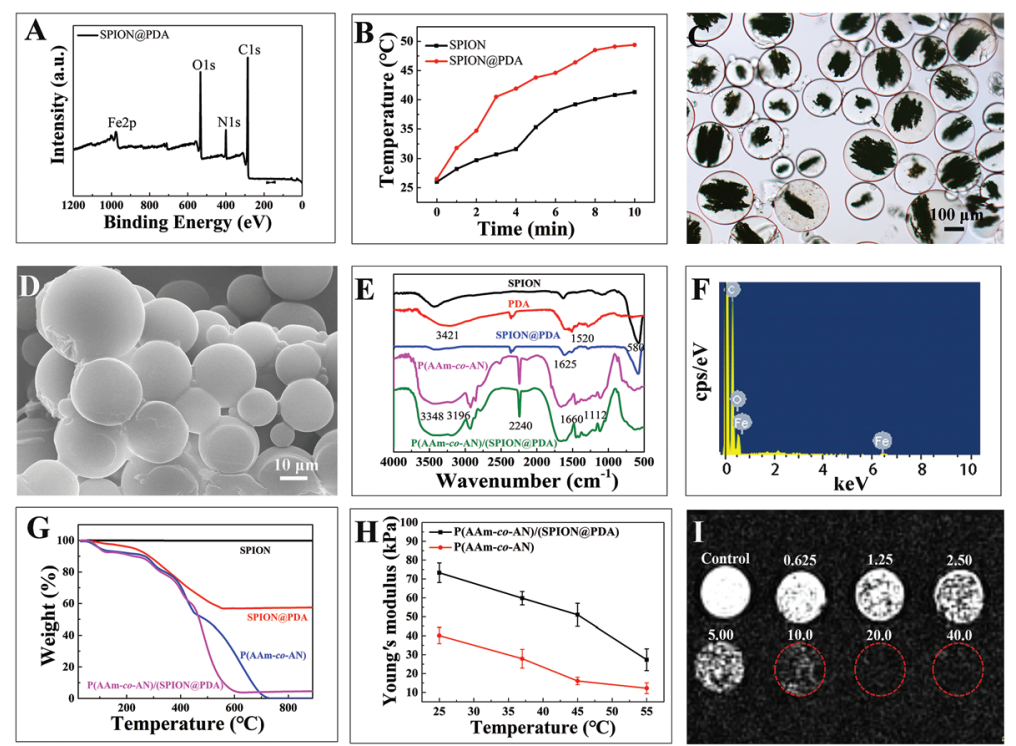

Fig. 2 Characterization studies of SPIONaPDA and P(AAm-co-AN)/(SPIONAPDA). (A) XPS spectrum of SPION@PDA. (B) Photothermal effect of SPION and SPIONAPDA. (C) Optical photograph, (D) SEM image, (F) SEM-EDS spectrum, and (H) the elasticity curve at different temperatures of P(AAm-co-AN)/(SPION@PDA). (E) FTIR spectra and (G) TGA curves of P(AAm-co-AN)/(SPION@PDA) and other substances. (I) $T_{2}$-Weighted MRI image of $\mathrm{P}(\mathrm{AAm}-\mathrm{CO}-\mathrm{AN}) /\left(\mathrm{SPION}(\mathrm{PDA})\right.$ with various concentrations $\left(\mathrm{mg} \mathrm{mL}^{-1}\right)$.

$1625 \mathrm{~cm}^{-1}$ corresponded to the surface-adsorbed water. The strong band at $2240 \mathrm{~cm}^{-1}$ in the FTIR spectra of the microspheres could be assigned to $\mathrm{C} \equiv \mathrm{N}$, suggesting the successful incorporation of the acrylonitrile moiety. The presence of the AAm moiety was confirmed by the broad stretching vibration absorption bands of amino at $3348 \mathrm{~cm}^{-1}$ and $3196 \mathrm{~cm}^{-1}$, as well as the stretching and bending vibration peaks of the $\mathrm{C}=\mathrm{O}$ group at $1660 \mathrm{~cm}^{-1}$ and $\mathrm{C}-\mathrm{N}$ at $1112 \mathrm{~cm}^{-1}$. There was no significant difference in the FTIR spectrum between $\mathrm{P}(\mathrm{AAm}-\mathrm{co}$ AN) and P(AAm-co-AN)/(SPION@PDA), indicating that the SPION@PDA was embedded inside the microspheres (Fig. 2E). The surface elementary analysis by SEM-EDS also confirmed the chemical composition of the $\mathrm{P}(\mathrm{AAm}-\mathrm{co}-\mathrm{AN})$ and $\mathrm{P}\left(\mathrm{AAm}-\mathrm{co}^{-}\right.$ AN)/(SPION@PDA) microspheres. In the SEM-EDS spectra, the presence of the $\mathrm{Fe}$ element should be originated from the SPION@PDA, and the presence of $\mathrm{C}$ and $\mathrm{O}$ elements mainly came from $\mathrm{P}(\mathrm{AAm}-\mathrm{Co}-\mathrm{AN})$ (Fig. $2 \mathrm{~F}$ and $\mathrm{S} 1 \mathrm{~F} \dagger)$. TGA was performed to further investigate the detailed composition of the P(AAm-co-AN)/(SPION@PDA). The content of PDA was found to be $40 \%$ in the SPION@PDA nanoparticles, and a 95.5\% weight loss was detected for P(AAm-co-AN)/(SPION@PDA) microspheres, suggesting that $7.5 \%(\mathrm{w} / \mathrm{w})$ of the SPION@PDA was incorporated into the P(AAm-co-AN)/(SPION@PDA) microspheres (Fig. 2G).

The mechanical property, in terms of elasticity, of the $\mathrm{P}(\mathrm{AAm}-c o-\mathrm{AN}) /(\mathrm{SPION} @ \mathrm{PDA})$ and P(AAm-co-AN) microspheres at different temperatures was also investigated by AFM. Due to the UCST nature of the microspheres, the Young's modulus of $\mathrm{P}(\mathrm{AAm}-\mathrm{co}-\mathrm{AN}) /(\mathrm{SPION} @ \mathrm{PDA})$ decreased along with an increase in temperature and the results were determined as $73.32 \mathrm{kPa}$ at $25{ }^{\circ} \mathrm{C}, 59.8 \mathrm{kPa}$ at $37{ }^{\circ} \mathrm{C}, 51.04 \mathrm{kPa}$ at $45^{\circ} \mathrm{C}$, and $27.34 \mathrm{kPa}$ at $55{ }^{\circ} \mathrm{C}$, respectively (Fig. $2 \mathrm{H}$ ). For $\mathrm{P}(\mathrm{AAm}-c o-\mathrm{AN})$ micro- spheres, the Young's moduli were $40.11 \mathrm{kPa}$ at $25{ }^{\circ} \mathrm{C}, 27.85$ $\mathrm{kPa}$ at $37{ }^{\circ} \mathrm{C}, 16.06 \mathrm{kPa}$ at $45{ }^{\circ} \mathrm{C}$, and $12.21 \mathrm{kPa}$ at $55{ }^{\circ} \mathrm{C}$, respectively. The Young's modulus of the microspheres increased after incorporating SPION@PDA nanoparticles and met the mechanical property requirements of embolic microspheres even at high temperature. ${ }^{24,29}$ Furthermore, the $T_{2}$ weighted MRI image showed a concentration-dependent contrast effect of P(AAm-co-AN)/(SPION@PDA) (Fig. 2I). The diameter and volume of the P(AAm-co-AN) microspheres presented a thermo-responsive pattern that it increased when temperature increased and returned to the original when temperature decreased, which should be attributed to the UCST properties (Fig. S1G $\dagger$ ). It is known that the UCST behavior could be attributed to the unique inter-intra-molecular hydrogen bonding and static charge interactions. These interactions become significantly weakened at increased temperature and consequently lead to the dissociation/dissolution of polymer chains in solvent. ${ }^{22-25}$ In the case of $\mathrm{P}(\mathrm{AAm}-\mathrm{co}-\mathrm{AN})$, hydrogen bonding is believed to play a major role in the volume-phase transition of the polymer chain. ${ }^{2-25}$ For the P(AAm-co-AN)-based microspheres, the UCST value of the crosslinked network, rather than the polymer chain, was found to be $40.4{ }^{\circ} \mathrm{C}$ as determined by DSC measurements.

Taken together, P(AAm-co-AN)/(SPION@PDA) microspheres were successfully synthesized. Additional characterization studies are available in the ESI. $\dagger$

\section{In vitro photothermal behaviors}

The temperature of the P(AAm-co-AN)/(SPION@PDA) microsphere suspension $\left(40 \mathrm{mg} \mathrm{mL}{ }^{-1}\right)$ increased from $26{ }^{\circ} \mathrm{C}$ to $83.5^{\circ} \mathrm{C}$ in 10 minutes with laser irradiation at a power density of $1.0 \mathrm{~W} \mathrm{~cm}^{-2}$, while those of the blank P(AAm-co-AN) micro- 

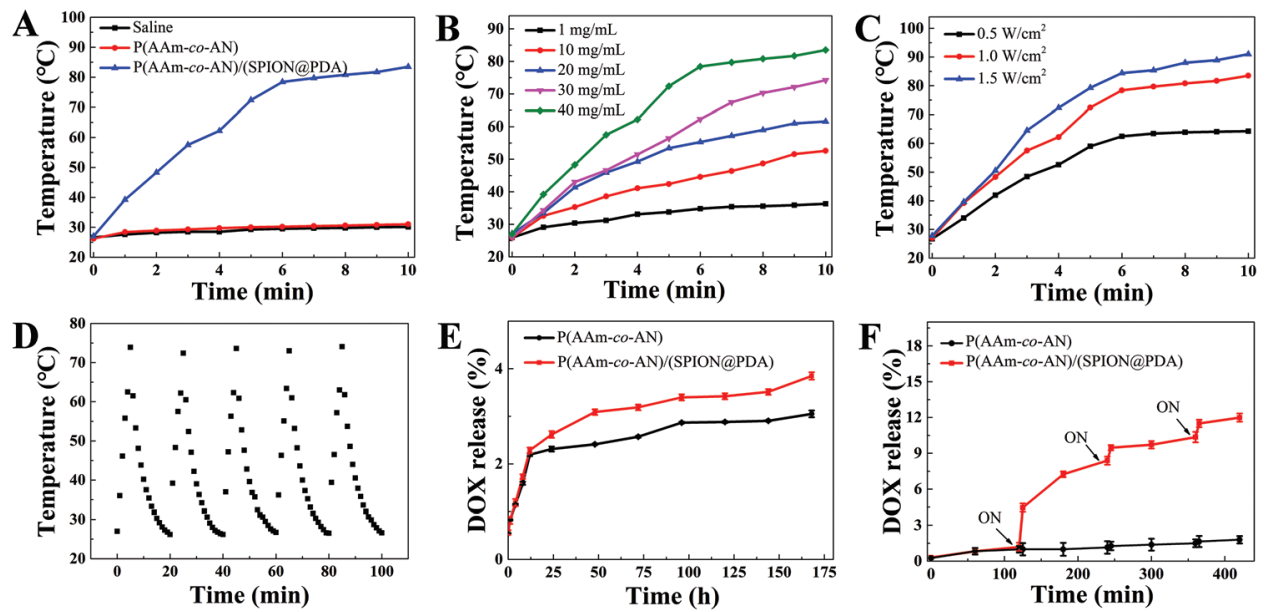

Fig. 3 In vitro photothermal efficiency, photothermal stability, and drug releasing profiles. Photothermal effects of P(AAm-co-AN)/(SPION@PDA) (A) compared to controls, (B) with different concentrations, and (C) with diverse irradiation powers. (D) Photothermal stability of P(AAm-co-AN)/ (SPION@PDA). Drug release profiles of P(AAm-CO-AN) and P(AAm-CO-AN)/(SPION@PDA) (E) without or (F) with laser irradiation.

spheres and saline merely increased from $26^{\circ} \mathrm{C}$ to $31.0^{\circ} \mathrm{C}$ and $30.1{ }^{\circ} \mathrm{C}$, respectively (Fig. 3A). Obviously, laser alone barely contributed to the photothermal effect, neither the $\mathrm{P}\left(\mathrm{AAm}-\mathrm{co}^{-}\right.$ AN) per se. The pronounced photothermal effect of the P(AAmco-AN)/(SPION@PDA) should be attributed mainly to the encapsulated SPION@PDA. The photothermal behavior of P(AAm-co-AN)/(SPION@PDA) microspheres showed a concentration-dependent pattern. The temperatures of the microspheres at various concentrations were found to be $36.3{ }^{\circ} \mathrm{C}$ for $1.0 \mathrm{mg} \mathrm{mL}{ }^{-1}, 52.6^{\circ} \mathrm{C}$ for $10 \mathrm{mg} \mathrm{mL}^{-1}, 61.6^{\circ} \mathrm{C}$ for $20 \mathrm{mg} \mathrm{mL}^{-1}$, $74.2{ }^{\circ} \mathrm{C}$ for $30 \mathrm{mg} \mathrm{mL}{ }^{-1}$, and $83.5{ }^{\circ} \mathrm{C}$ for $40 \mathrm{mg} \mathrm{mL}^{-1}$ after being irradiated at a power density of $1.0 \mathrm{~W} \mathrm{~cm}^{-2}$ for 10 minutes (Fig. 3B). Likewise, the rate and magnitude of temperature elevation increased at a higher NIR power level. The temperatures increased from $26^{\circ} \mathrm{C}$ to $64.2{ }^{\circ} \mathrm{C}, 83.5^{\circ} \mathrm{C}$, and $91.0^{\circ} \mathrm{C}$ with laser irradiation for 10 minutes at power densities $0.5 \mathrm{~W} \mathrm{~cm}^{-2}, 1.0 \mathrm{~W} \mathrm{~cm}^{-2}$, and $1.5 \mathrm{~W} \mathrm{~cm}^{-2}$, respectively (Fig. 3C). Noteworthily, the hyperthermia effect yielded by $\mathrm{P}(\mathrm{AAm}-\mathrm{co}-\mathrm{AN}) /(\mathrm{SPION} @ \mathrm{PDA})$ microspheres surpassed the proven requirement for eliminating tumor cells. ${ }^{19}$

Furthermore, heating-cooling cycles were performed to evaluate the photothermal stability of the $\mathrm{P}(\mathrm{AAm}-\mathrm{co}-\mathrm{AN}) /$ (SPION@PDA) microspheres under repeated NIR irradiation. It was found that the photothermal effect of the microsphere remained constant after five cycles of "ON" and "OFF" switch of NIR irradiation (Fig. 3D). The result clearly demonstrated that the P(AAm-co-AN)/(SPION@PDA) remained intact even after several cycles of laser-triggered phase transition. ${ }^{24}$

In brief, the $\mathrm{P}(\mathrm{AAm}-\mathrm{co}$-AN)/(SPION@PDA) microsphere presented advantages including superior photothermal conversion efficiency as well as good photothermal stability, providing great potential for the photothermal treatment of cancer.

SPION is capable of generating hyperthermia when exposed to near infrared laser or alternating magnetic field, namely photothermal and magnetic hyperthermal (MHT) effect, respectively. ${ }^{30,31}$ In this proof-of-concept study, the photo- thermal effect was selected due to a relatively higher efficiency compared with MHT. ${ }^{30,31}$ Polydopamine was coated onto SPION to further amplify the photothermal conversion efficiency. ${ }^{32}$ Consequently, the microspheres exhibited potent photothermal effect. Furthermore, the UCST property endows the microspheres with a photothermal-triggered drug release pattern, facilitating the contemporaneous presence of thermaland chemo-therapy which may aid in the synergistic enhancement of individual treatment modality.

\section{Drug loading and release profiles}

In this study, the drug loading efficiencies of $\mathrm{P}(\mathrm{AAm}-c o-\mathrm{AN})$ and P(AAm-co-AN)/(SPION@PDA) were found to be $2.68 \pm 0.5 \%$ and $2.01 \pm 0.7 \%(\mathrm{w} / \mathrm{w})$, respectively (Fig. S1 $\dagger$ ), which were comparable to the loading capacity of commercial microspheres. ${ }^{9,10}$ As platforms designed with a thermal-triggered release property, the cumulative release of DOX from $\mathrm{P}(\mathrm{AAm}-\mathrm{co}-\mathrm{AN})$ and $\mathrm{P}(\mathrm{AAm}-c o-\mathrm{AN}) /(\mathrm{SPION} @ \mathrm{PDA})$ microspheres at $37{ }^{\circ} \mathrm{C}$ after 7 days were only $3.85 \%$ and $3.05 \%$, respectively, indicating the good storage stability of these drug-loaded microspheres (Fig. 3E). On the other hand, the accelerated release upon NIR irradiation was observed that approximately $4.45 \%$ of DOX was offloaded from P(AAm-co-AN)/ (SPION@PDA) microspheres after the first NIR irradiation, and $11.5 \%$ of DOX was released cumulatively subsequent to additional two irradiation processes (Fig. $3 \mathrm{~F}$ ). In contrast, only $1.64 \%$ of DOX released from P(AAm-co-AN) was subjected to the identical procedure (Fig. 3F). The underlying mechanism of such phenomena could be attributed to the USCT nature and photothermal feature of the P(AAm-co-AN)/(SPION@PDA) microspheres. At temperature lower than the UCST of the microspheres, DOX was tightly entrapped in the microspheres, and the diffusion of DOX was hindered by the strong intermolecular interactions between DOX and the microsphere matrix. When NIR laser was given, the $\mathrm{P}(\mathrm{AAm}-c o-\mathrm{AN}) /$ (SPION@PDA) microspheres showed photothermal conversion, 
and the microsphere matrix underwent phase transition upon increased temperature and became more hydrophilic. The crosslink density decreased apparently as evidenced by the larger diameter of the microspheres at higher temperature (Fig. S1G $\dagger$ ), leading to a significantly accelerated release of DOX from the matrix. Meanwhile, the diffusion of DOX was enhanced at increased temperature as well. For P(AAm-co-AN)/(SPION@PDA) microspheres in the absence of NIR irradiation or P(AAm-co-AN) microspheres without photothermal conversion capability, no remarkable changes were observed with respect to the release behaviors of DOX. Given the potent chemosensitization effect of hyperthermia, the contemporaneous presence of thermal- and chemo-therapy was supposed to exert a significant synergistic interaction, which was further investigated and confirmed in the following in vitro and in vivo studies.

\section{Biocompatibility}

The biocompatibility of the $\mathrm{P}(\mathrm{AAm}-\mathrm{co}-\mathrm{AN})$ and $\mathrm{P}(\mathrm{AAm}-\mathrm{co}-\mathrm{AN}) /$ (SPION@PDA) microspheres was evaluated by cytotoxicity and hemolysis assays. The viabilities of the tested cells, including BEL-7402, L02, and HepG2 cells, remained over 80\% after co-culturing with extracts prepared from microsphere concentrations up to $200 \mathrm{mg} \mathrm{mL}^{-1}$ (Fig. 4A). Furthermore, the hemolysis ratios were less than $5.0 \%$, demonstrating excellent hemocompatibility of these microspheres (Fig. 4B). The cytotoxicity and hemolysis assays proved the satisfactory biocompatibility of the P(AAm-co$\mathrm{AN})$ and P(AAm-co-AN)/(SPION@PDA) microspheres and granted further studies for their applications in vivo.

\section{In vitro anti-tumor assays}

Cellular assays were performed to assess the anti-tumor potency of the microspheres, especially to explore the synergis-
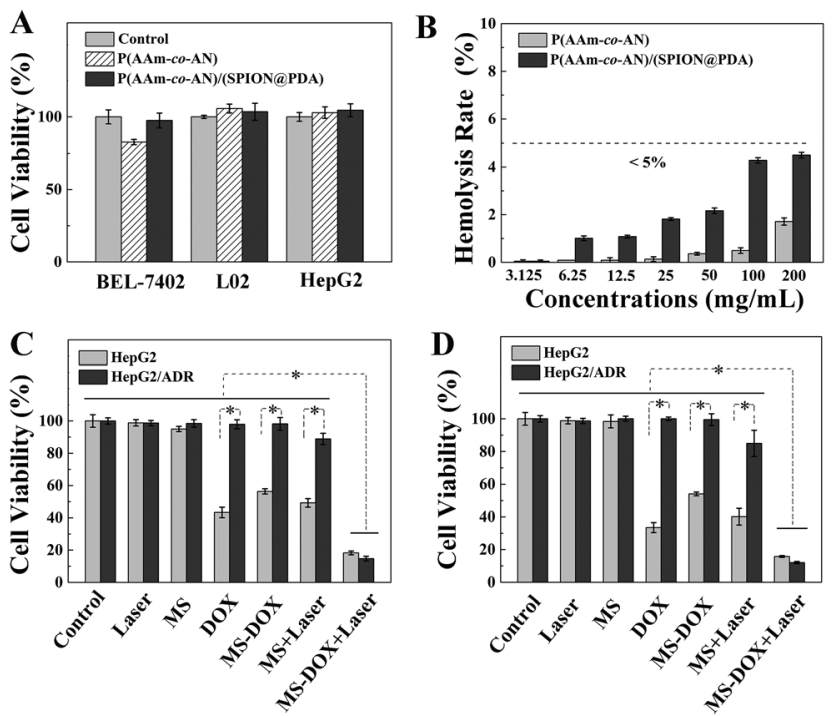

Fig. 4 Biocompatibility assessment and in vitro anti-tumor activity. (A) Cytotoxicity and (B) hemolysis analysis of $\mathrm{P}(\mathrm{AAm}-\mathrm{CO}-\mathrm{AN})$ and $\mathrm{P}(\mathrm{AAm}-$ co-AN)/(SPION@PDA). Cytotoxicity at DOX and microsphere concentrations of (C) $1.0 \mu \mathrm{g} \mathrm{mL}^{-1}$ and $0.5 \mathrm{mg} \mathrm{mL}^{-1}$, and (D) $2.0 \mu \mathrm{g} \mathrm{mL}^{-1}$ and $1.0 \mathrm{mg} \mathrm{mL}^{-1}$, respectively, ${ }^{*} p<0.05$. tic effect of photothermal therapy and chemotherapy against chemoresistant HepG2/ADR cells. There was no apparent cytotoxicity for tumor cells subjected to either NIR laser or microsphere treatment, suggesting that the NIR laser or microspheres exerted no therapeutic effect by themselves. The viabilities of HepG2 and HepG2/ADR cells treated by free DOX of $1.0 \mu \mathrm{g} \mathrm{mL} \mathrm{mL}^{-1}$ were $43.3 \%$ and $97.8 \%(p<0.05)$, respectively, demonstrating that the chemoresistant nature of HepG2/ADR cells remarkably impaired the effectiveness of chemotherapy (Fig. 4C). Likewise, the viabilities of HepG2 and HepG2/ADR cells subject to $0.5 \mathrm{mg} \mathrm{mL} \mathrm{mL}^{-1}$ of DOX loaded P(AAm-co-AN)/ (SPION@PDA) were 56.3\% and 98.2\% $(p<0.05)$, respectively, both of which were slightly higher compared with the cellular viabilities of the free DOX group (Fig. 4C). It was due to the restrained release of DOX from the microspheres in the absence of NIR irradiation (Fig. 3E). Hyperthermia induced by the treatment of $\mathrm{P}(\mathrm{AAm}-\mathrm{co}-\mathrm{AN}) /(\mathrm{SPION} @ \mathrm{PDA})$ microspheres with NIR irradiation resulted in decreased viabilities to $49.2 \%$ for HepG2 and 88.9\% for HepG2/ADR cells ( $p<0.05)$, confirming the anti-tumor effect of thermotherapy (Fig. 4C). On the other hand, it is known that chemoresistance is usually concomitant with thermotolerance which is at least partially due to the activation of heat shock proteins (HSPs). ${ }^{33}$ According to the previous report, HepG2/ADR cells express the high level of HSPs, ${ }^{34}$ which might be responsible for the findings in this work that HepG2/ADR cells are less sensitive to either chemoor thermo-therapy. Strikingly, only $18.3 \%$ HepG2 and $14.7 \%$ HepG2/ADR cells remained viable after treatment of DOX loaded P(AAm-co-AN)/(SPION@PDA) with NIR irradiation $(p<$ 0.05 compared to all other groups), indicating that the combination of chemotherapy and thermotherapy substantially enhanced anti-tumor response when compared with the individual treatment modality (Fig. 4C). Similar outcomes were observed in another series of experiments, in which the concentrations of DOX and the microspheres were set at $2.0 \mu \mathrm{g}$ $\mathrm{mL}^{-1}$ and $1.0 \mathrm{mg} \mathrm{mL}^{-1}$, respectively. The viabilities of HepG2 and HepG2/ADR were $98.8 \%$ and $98.7 \%$ in the Laser group, $98.4 \%$ and $100 \%$ in the MS group, $33.4 \%$ and $100 \%$ in the DOX group $(p<0.05), 54.1 \%$ and $99.5 \%$ in the MS-DOX group $(p<0.05), 40.1 \%$ and $85.0 \%$ in the MS + Laser group $(p<$ 0.05 ), and $15.7 \%$ and $12.1 \%$ in the MS-DOX + Laser group ( $p<$ 0.05 compared to all other groups), respectively (Fig. 4D).

The in vitro anti-tumor studies confirmed the hypothesis that simultaneous photothermal therapy and chemotherapy yielded considerable synergistic effect, particularly for chemoresistant tumor cells.

\section{Rabbit VX2 liver tumor models and in vivo experiments}

A rabbit VX2 liver tumor model is a standard animal model for liver cancer-related research, especially for evaluating novel interventional technology, facilities, and treatment strategies. Despite the distinct cellular origin of VX2 cells, there are several advantages of this model that the biological behaviors and imaging characters are very similar to human liver cancer, the operative procedure for establishment is simple with high success rate, and the tumor grows rapidly. ${ }^{7}$ Furthermore, 
rabbit vascular lumens are large enough to perform endovascular procedures with a clinically used catheter. In this work, rabbit VX2 liver tumor models were successfully established for in vivo experiments as demonstrated by the imaging surveillance that tumor mass with a diameter of approximately $1.5 \mathrm{~cm}$ developed in the left liver lobe 2-3 weeks after implantation. The VX2 tumor exhibited typical hypervascular imaging features similar to human liver cancer in contrast-enhanced CT images that it presented as a low-density lesion on plain scan, a ring-shape enhancement with a non-enhanced core area on the hepatic arterial phase, and showed relatively lower density on the portal venous phase compared to normal liver (Fig. 6). During the TACE procedure, digital subtraction angiography (DSA) of the celiac artery, hepatic artery, and hepatic lobar or segmental artery was performed separately to clarify the vascular anatomy and the tumor feeding artery. Angiographic images revealed the enlarged and distorted tumor vasculatures in the left hepatic lobe. TACE was carried out successfully and satisfactory embolization was achieved in $90.1 \%(10 / 11)$ of the intent-to-treat rabbits. One rabbit in the treatment group did not achieve completed embolization due to collateral circulations between tumor vessels and important normal arteries (Fig. S2 $\dagger$ ). Repeated DSA images after emboli- zation showed that the blood feeding of the tumor was substantially deprived (Fig. 5A and B). One rabbit was employed for evaluating the in vivo MRI contrast effect of the $\mathrm{P}\left(\mathrm{AAm}-\mathrm{Co}^{-}\right.$ AN)/(SPION@PDA) microsphere and the MRI scanning was conducted before and after TACE. The results revealed a $46.2 \%$ decrease in MRI signal intensity in tumor lesions after embolization $(191.22 \pm 15.88$ versus $102.854 \pm 12.97, n=3, p<0.05$, Fig. 5C-E), indicating the potential of the microsphere as an MRI contrast agent for monitoring its distribution. After embolization, two rabbits were selected to confirm the expected photothermal effect of the P(AAm-co-AN)/(SPION@PDA) microsphere in vivo. It was found that the average temperatures of the peripheral and core regions of the tumor reached over $61.5{ }^{\circ} \mathrm{C}$ and $66.9^{\circ} \mathrm{C}$ in 10 minutes, respectively, which far exceeded the threshold for ablation ${ }^{19}$ and a robust tumor-eliminating effect can be expected using this therapeutic strategy (Fig. 5F).

All of the 8 rabbits in the treatment group were subjected to laser irradiation right after TACE. Treatment procedures were completed in all of the experimental animals. The average temperatures of the peripheral tumor region as recorded and measured using an infrared thermal camera increased from $35.6 \pm 1.2^{\circ} \mathrm{C}$ to $78.0 \pm 5.4{ }^{\circ} \mathrm{C}$ in 10 minutes (Fig. $5 \mathrm{G}$ and $\mathrm{H}$ ).
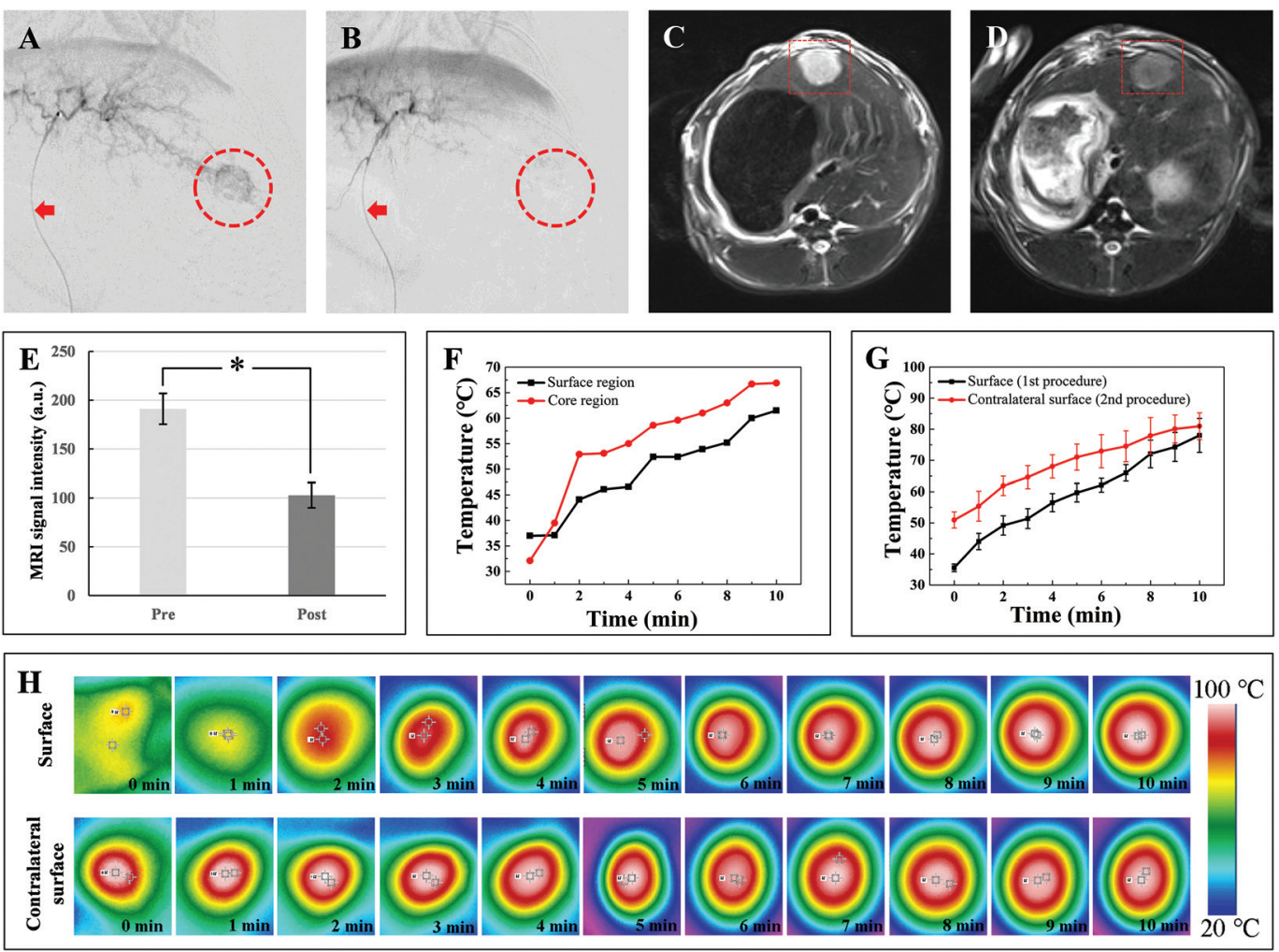

Fig. 5 In vivo experiments. Digital subtraction angiography (DSA) images (A) before and (B) after TACE. Red dotted lines showed the vascularity of the tumor, and red arrows indicated the catheter. Exemplary $T_{2}$-weighted MRI images of tumor (C) before and (D) after TACE using P(AAm-Co-AN)/ (SPION@PDA) microspheres. Red dotted squares indicated tumor locations. (E) MRI signal intensity before and after TACE, ${ }^{*} p<0.05$. (F) Temperatures of the surface and core regions of tumor mass after TACE and laser irradiation as determined using a thermocouple thermometer. (G) Average temperatures of tumor surface and contralateral tumor surface during two sequential laser irradiation procedures as measured using an infrared thermal camera in all of the 8 rabbits in the treatment groups. $(\mathrm{H})$ Representative thermal scanning images of the tumor surface and contralateral tumor surface exposed to laser irradiation at different time points after TACE. 
When initiating the second laser irradiation procedure to the contralateral surface of tumor mass, the temperature recorded using the infrared thermal camera was $50.9 \pm 2.6^{\circ} \mathrm{C}$, which was sufficient for thermotherapy, further confirming the feasibility of the proposed strategy. During the second procedure of laser irradiation, the temperatures increased from $50.9 \pm$ $2.6{ }^{\circ} \mathrm{C}$ to $81.0 \pm 4.3^{\circ} \mathrm{C}$ (Fig. $5 \mathrm{G}$ and $\mathrm{H}$ ).

\section{Therapeutic efficacy}

The therapeutic efficacy was evaluated by imaging studies according to mRECIST criteria. ${ }^{26}$ One week after treatment, CR was observed in all of the 4 rabbits (Fig. 6B and D). Two weeks after treatment, $\mathrm{CR}$ and partial response (PR) were $75 \%(3 / 4)$ and $25 \%(1 / 4)$, respectively (Fig. 6C and D). In contrast, all tumors in the control group progressed significantly and were evaluated as progressive disease (PD) (Fig. 6A and D). Overall, TACE plus laser irradiation achieved a response of $87.5 \% \mathrm{CR}$ and $12.5 \%$ PR. The average changes of the longest viable tumor diameters for the treatment group and the control group were $-91.5 \%$ and $+86.5 \%$, respectively, compared to the baseline (Fig. 6D and Table $\mathrm{S} 1 \dagger$ ). As expected, the tumor with insufficient delivery of microspheres and embolization resulted in an unfa- vorable outcome (PR and $-32.3 \%$ in the longest viable tumor diameter) compared to those with completed devascularization (CR and $-100 \%$ ), further confirming the therapeutic activity of the microspheres and the fundamental role of embolization in treating liver cancer (Fig. 6 and S2, Table S1†). Complete response, which is defined as no residual enhancement in tumor mass in the contrast-enhanced imaging study, is fairly difficult to attain in the clinical management of liver cancer. ${ }^{26}$ It was reported that one TACE procedure yields a $52.5 \%$ CR rate even in selected patients with relatively small tumor lesions and preserved liver function, and a CR rate of $67.2 \%$ was finally achieved after multiple TACE treatments. ${ }^{11}$ More importantly, it was found that the achievement of complete response at the first TACE procedure remained the most robust positive predictor for patient survival, emphasizing the importance of pursuing the superior early treatment response. ${ }^{11}$ Herein, a single TACE plus laser irradiation procedure attained an impressive outcome (87.5\% of CR), giving the great potential of the developed microsphere for improving prognosis of patients with liver cancer.

In this study, pathological examinations were performed to further investigate the therapeutic efficacy of the novel treatment strategy. Massive necrosis was observed in treated
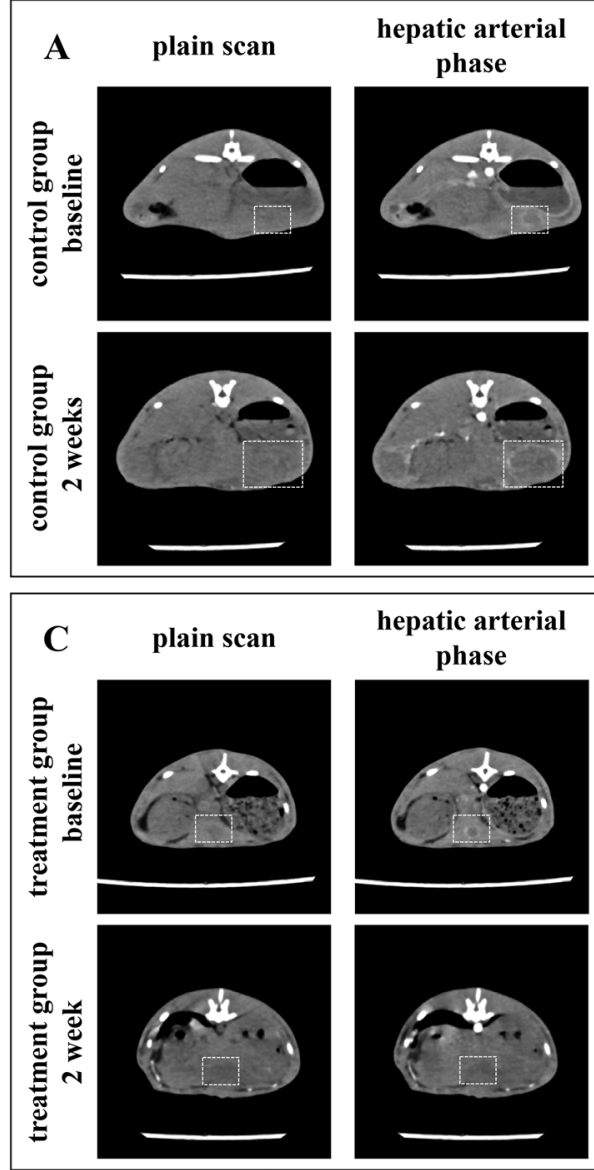
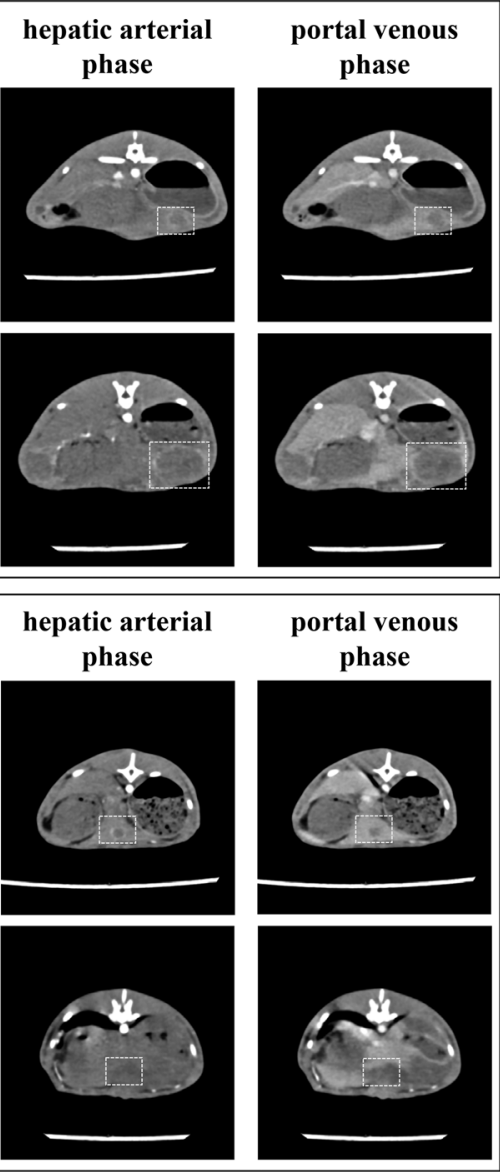
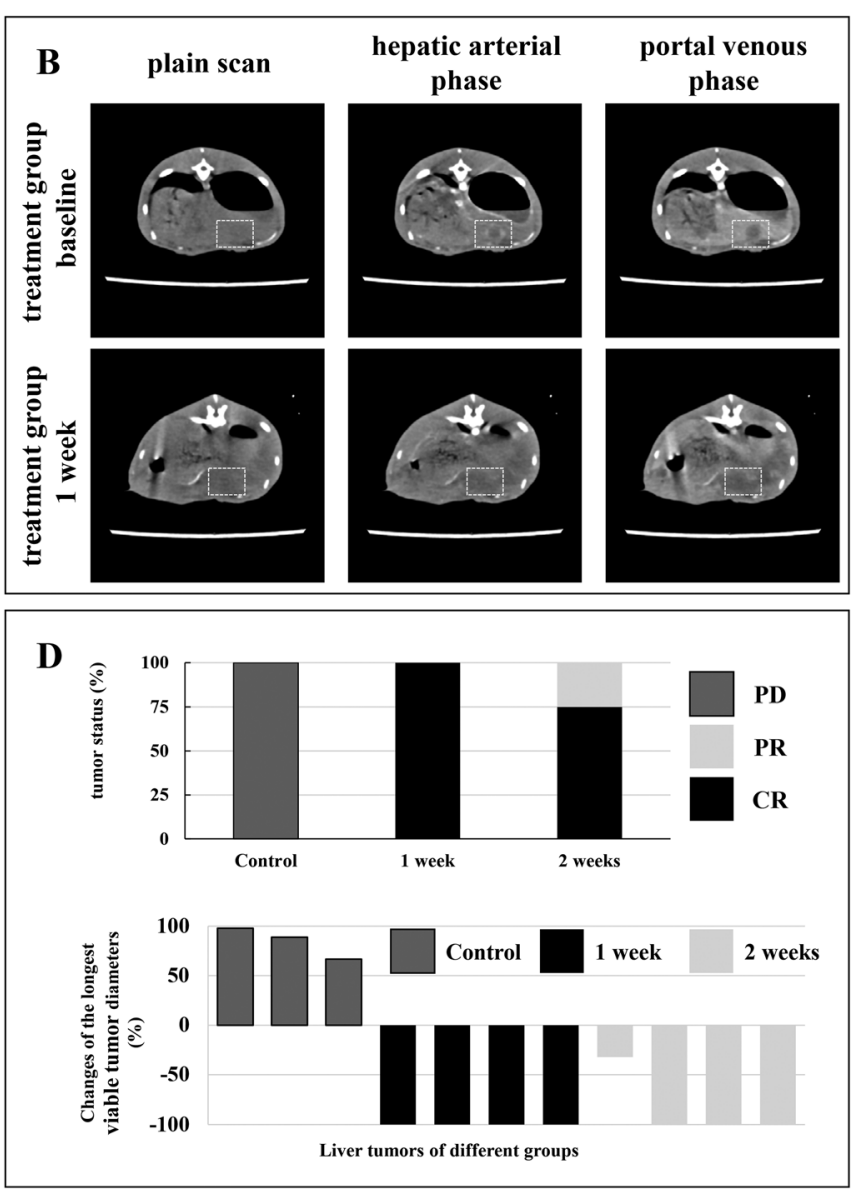

Fig. 6 Therapeutic efficacy. Representative dual-phase dynamic contrast-enhanced CT images of rabbit VX2 liver tumors of (A) the control group and (B and C) treatment groups at different time points. White dotted squares indicated tumor locations. (D) Treatment response and the changes of the longest viable tumor diameters of control and treatment groups at different time points compared to the baseline. CR, complete response; PR, partial response; PD, progressive disease. 
tumors and embolized microspheres can be viewed within a tumor vascular lumen surrounded by necrotic tumor tissue (Fig. 7B, C and E). Pathology-proven tumor necrotic rates were $90.4 \pm 11.1 \%$ and $84.0 \pm 13.0 \%$ at 1 and 2 weeks after treatment, respectively, and complete necrosis with no residual viable tumor after treatment was found in $37.5 \%(3 / 8)$ of the treated tumors. Again, the pathological necrotic ratio of the tumor without complete embolization was merely $68.6 \%$, which was at the same magnitude as that of the control group (Fig. 7 and $\mathrm{S} 2 \dagger$ ). Of note, $65.2 \pm 18.1 \%$ necrotic rate for tumors of the control group was observed and the necrosis occurred mainly in the core region (Fig. 7A and D). This phenomenon was due to the relatively insufficient blood supply in the core area secondary to the fast-growing nature of the VX2 tumor cells, resulting in a baseline level of tumor necrosis. The malignant cells in the peripheral area are primarily responsible for rapid proliferation and invasion of the tumor. Elimination of these tumor cells is vitally important for achieving good therapeutic efficacy. Pronounced necrosis in the peripheral area of the tumor lesion after treatment can be observed, which suggested the achievement of robust tumorsuppressive outcome (Fig. 7B and C). On the other hand, the tumor cells in the marginal area of the control group remained intact, maintaining the capability of rapid and constant proliferation (Fig. 7A). In total, the average tumor necrotic rate of the treatment group was $87.2 \pm 11.7 \%$, which significantly surpassed that of the control group $(65.2 \pm 18.1 \%, p<0.05$, Fig. 7D).

The major aim of TACE is to achieve pathological complete necrosis of the tumor lesion. Previous studies have reported that the extent of tumor necrosis correlated with prognosis. ${ }^{12,14}$ However, it remains challenging for TACE per se to eliminate tumor cells entirely and the residual viable tumor cells are the important sources of rapid recurrence and tumor progression that remarkably deteriorate the patient prognosis. In a large-scale study analyzing over 3000 cases, the pathologically proved complete necrosis can only be achieved in $22.4 \%$ of the patients who received TACE treatment alone; and even for patients subjected to combined therapy of TACE and thermal ablation, pathological $\mathrm{CR}$ rate merely increased to $26.0 \% .^{15}$ Furthermore, it is much more difficult to achieve pathological complete necrosis compared to imaging criteria, and previous studies reported that residual tumor cells can be found in most (89\%) of the lesions which were determined as CR in imaging analysis. ${ }^{12-14}$ Therefore, it is critical to explore novel strategies for enhancing liver tumor eradication.

In this work, photothermal therapy was introduced as an additional modality for combined treatment. Thermotherapy is effective for the treatment of cancers that a moderately elevated temperature of $39-45{ }^{\circ} \mathrm{C}$ is sufficient to destroy tumor cells. ${ }^{19}$ Given the inherent chemoresistant nature of liver cancer, ${ }^{4}$ the capability of thermotherapy to serve as a chemosensitizer is vitally helpful. This was proved in the cellular anti-cancer experiment in this study. Besides, UCSTtype characteristic gives the microsphere the ability of exerting the photothermal effect and drug releasing simultaneously to maximize the synergistic interaction of these two modalities. In consequence, $87.2 \%$ pathologically proven tumor necrotic rate and $37.5 \%$ CR were achieved. The robust tumor-eliminating results observed in this study demonstrated the effectiveness of combining TACE and photothermal therapy, implying great potential of the novel microsphere as a potent tool for enhancing the treatment response of liver cancer.
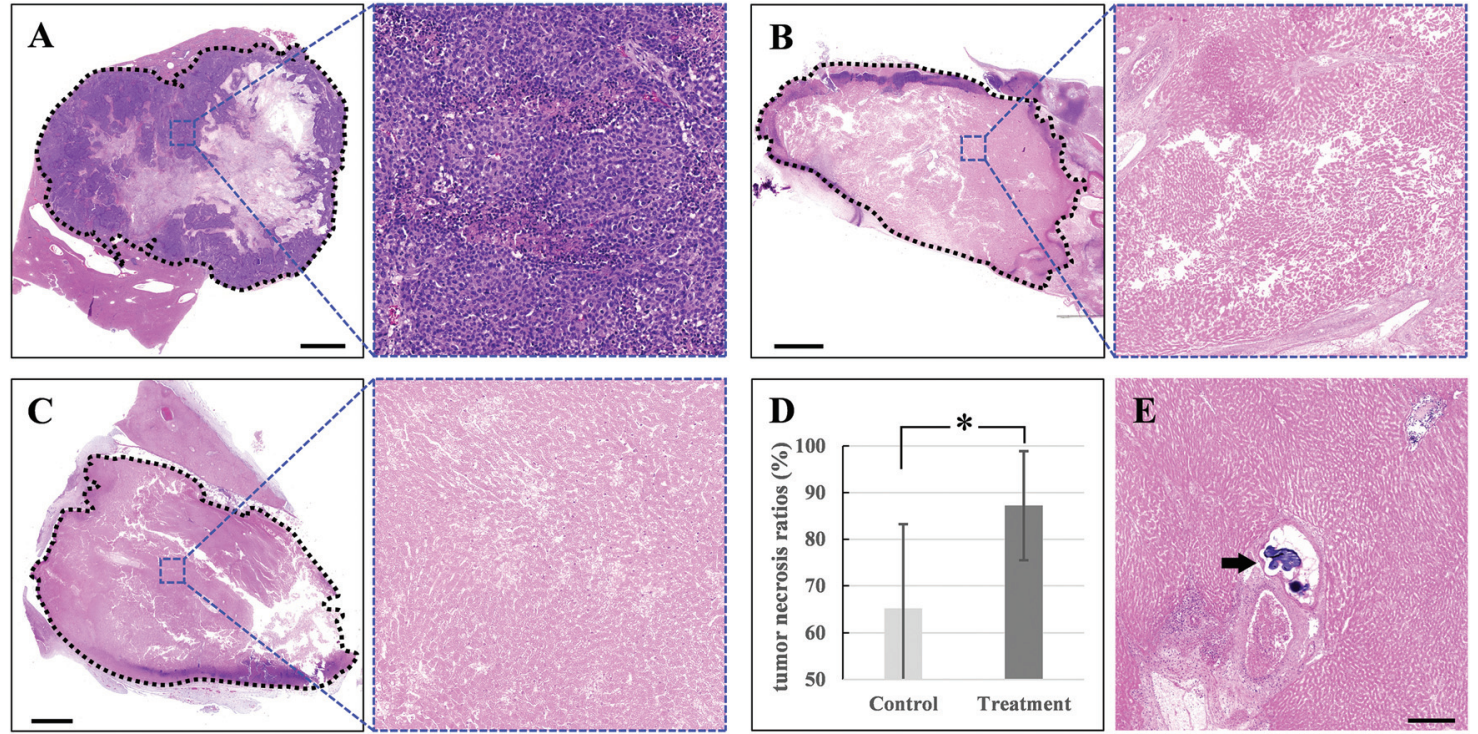

Fig. 7 Pathological examinations of tumor mass of control and treatment groups. Representative HE staining and the corresponding high-magnification images of tumors of (A) the control group 2 weeks after the baseline, and treatment groups (B) 1 week and (C) 2 weeks after treatment; dotted lines delineated the tumor mass. (D) Tumor necrotic ratios of control and treatment groups, * $p<0.05$. (E) Exemplary images of the microspheres which embolized within the tumor vascular lumen in necrotic tumor mass (black arrow). Scale bars: $2.5 \mathrm{~mm}$ in (A)-(C); $250 \mu \mathrm{m}$ in (E). 

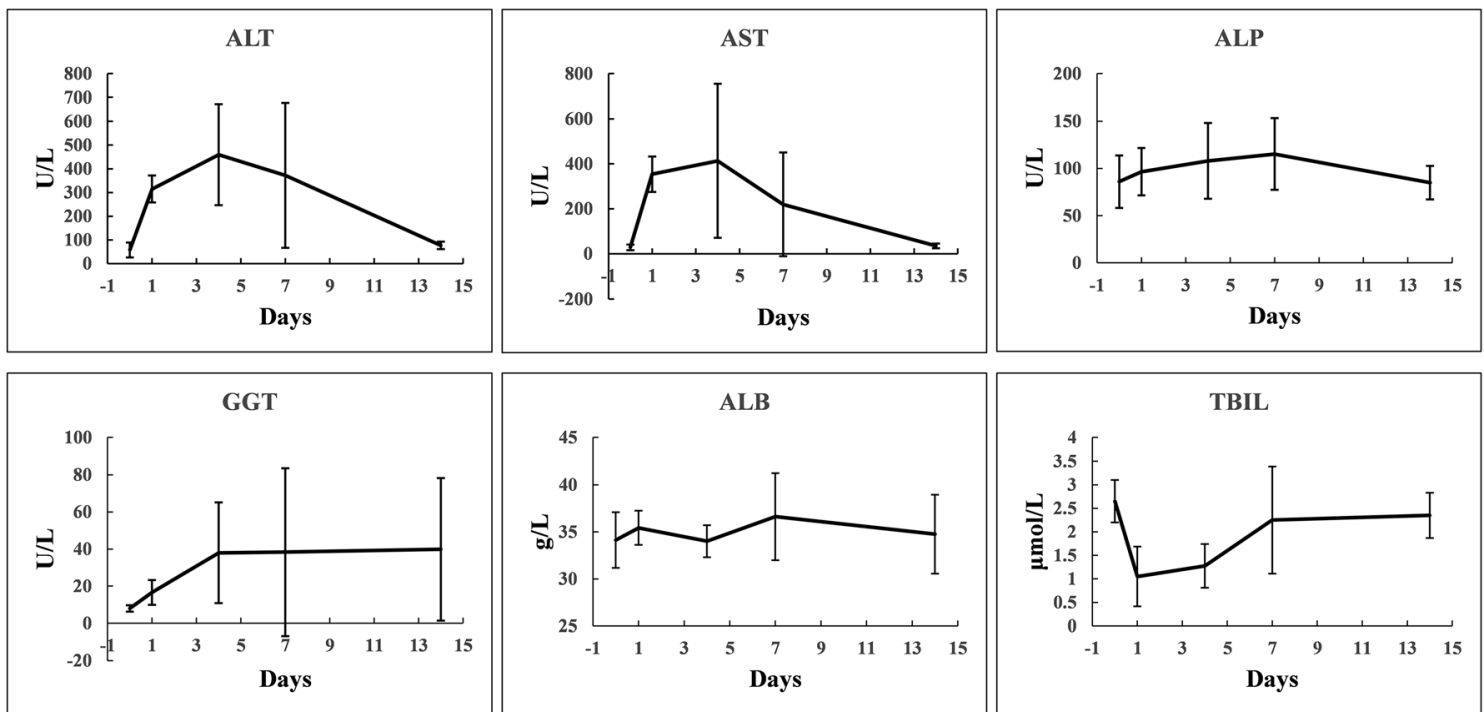

Fig. 8 Therapeutic safety as assessed by liver enzyme and biochemical examinations. Serum concentrations of alanine aminotransferase (ALT, $U$ $\mathrm{L}^{-1}$ ), aspartate aminotransferase (AST, $\cup \mathrm{L}^{-1}$ ), alkaline phosphatase (ALP, $\left.\mathrm{U} \mathrm{L}^{-1}\right)$, gamma glutamyl transpeptidase $\left(\mathrm{GGT}, \mathrm{U} \mathrm{L}^{-1}\right)$, albumin $\left(A L B, \mathrm{~L}^{-1}\right)$, and total bilirubin (TBIL, $\mu \mathrm{mol} \mathrm{L}^{-1}$ ) before and after treatment. $p>0.05$ for day 0 versus day 14 in all examinations.

\section{Therapeutic safety}

TACE treatment is usually accompanied by injury of normal liver which manifests as an increase in serum concentrations of liver enzymes and impaired liver synthetic and metabolic functions. Furthermore, the vast majority of liver cancer patients comorbid with chronic liver disease and even decompensated cirrhosis, which is also a key determinant of clinical decision-making. Therefore, aside from therapeutic efficacy, the safety evaluation of a novel treatment strategy for liver cancer is critically important. In this study, the treatment safety was determined by liver enzymes and biochemical examinations employing parameters including ALT, AST, ALP, GGT, ALB, and TBIL. It was found that the level of serum liver enzymes elevated after treatment but recovered subsequently within two weeks. No changes in serum concentrations of ALB and TBIL were observed (Fig. 8 and Table $\mathrm{S} 2 \dagger$ ). These findings proved the safeness of the novel approach for treating liver cancer.

\section{Conclusions}

In summary, a novel versatile UCST-type composite microsphere was successfully prepared for simultaneous chemoembolization and photothermal therapy. The microspheres were uniform in morphology with a diameter of 100-300 $\mu \mathrm{m}$ and exhibited favorable biocompatibility, drug loading capability, and photothermal-triggered release pattern as well as strong photothermal effect. Cellular anti-tumor tests demonstrated a remarkably synergistic enhancement of photothermal therapy and chemotherapy against chemoresistant liver cancer cells. The in vivo therapeutic efficacy and safety evaluations were implemented using rabbit VX2 liver tumor models. It was revealed that a single procedure of TACE and photothermal therapy resulted in $87.5 \%$ complete response and $12.5 \%$ partial response for the treatment group, whereas all tumors in the control group progressed significantly. Imaging examinations using contrast-enhanced CT scanning indicated that the viable tumor diameter decreased by $91.5 \%$ after treatment, while that in the control group increased by $86.5 \%$. The pathology-proven tumor necrotic rate was $87.2 \%$, which significantly surpassed that of $65.2 \%$ in the control group. Furthermore, serum liver enzyme and biochemical studies revealed a temporary liver injury, which can be fully recovered. Our findings demonstrated that this microsphere may provide advantages for improving therapeutic efficacy of TACE against liver cancer.

\section{Author contributions}

Conception, design, and study supervision: Chao Zhang, Run Lin, and Weiling He; Funding support: Jianyong Yang, Chao Zhang, Run Lin, and Weiling He; Acquisition of data: Dan Huang, Haitao Dai, Keyu Tang, Bin Chen, Hongzhang Zhu, Dubo Chen, Nan Li, Yingzhao Wang, Chuwei Liu, and Yonghui Huang; Analysis and interpretation of data: Dan Huang, Haitao Dai, Keyu Tang, Jianyong Yang, Chao Zhang, Run Lin, and Weiling He; Writing, review, and/or revision of the manuscript: Dan Huang, Haitao Dai, Keyu Tang, Chao Zhang, Run Lin, and Weiling He.

\section{Conflicts of interest}

There are no conflicts of interest to declare. 


\section{Acknowledgements}

The authors thank Mr Li Gong at the Instrumental Analysis and Research Centre, Sun Yat-sen University, for his assistance in AFM. This work was supported by the National Natural Science Foundation of China (81671792, 81701834, 81760322, 81871994, and 81971760), the Guangdong Provincial Natural Science Foundation for Distinguished Young Scholars (2019B151502063), the Guangdong Natural Science Foundation (2018A030310285), the Science and Technology Planning Project of Guangdong Province (2015B010125004), the Guangzhou Science and Technology Planning Program (201902020018 and 201904010398), and the Fundamental Research Funds for the Central Universities (18ykzd04).

\section{References}

1 F. Bray, J. Ferlay, I. Soerjomataram, R. L. Siegel, L. A. Torre and A. Jemal, Ca-Cancer J. Clin., 2018, 68, 394-424.

2 A. Villanueva, N. Engl. J. Med., 2019, 380, 1450-1462.

3 R. L. Siegel, K. D. Miller and A. Jemal, Ca-Cancer J. Clin., 2019, 69, 7-34.

4 A. Forner, M. Reig and J. Bruix, Lancet, 2018, 391, 13011314.

5 J. L. Raoul, A. Forner, L. Bolondi, T. T. Cheung, R. Kloeckner and T. de Baere, Cancer Treat. Rev., 2019, 72, 28-36.

6 W. Sieghart, F. Hucke and M. Peck-Radosavljevic, J. Hepatol., 2015, 62, 1187-1195.

7 R. J. Lewandowski, J. F. Geschwind, E. Liapi and R. Salem, Radiology, 2011, 259, 641-657.

8 M. Caine, D. Carugo, X. Zhang, M. Hill, M. R. Dreher and A. L. Lewis, Adv. Healthcare Mater., 2017, 6, 1601291.

9 J. Hu, H. Albadawi, B. W. Chong, A. R. Deipolyi, R. A. Sheth, A. Khademhosseini and R. Oklu, Adv. Mater., 2019, 31, e1901071.

10 K. Fuchs, R. Duran, A. Denys, P. E. Bize, G. Borchard and O. Jordan, J. Controlled Release, 2017, 262, 127-138.

11 B. K. Kim, S. U. Kim, K. A. Kim, Y. E. Chung, M. J. Kim, M. S. Park, J. Y. Park, D. Y. Kim, S. H. Ahn, M. D. Kim, S. I. Park, J. Y. Won, D. Y. Lee and K. H. Han, J. Hepatol., 2015, 62, 1304-1310.

12 M. A. Allard, M. Sebagh, A. Ruiz, C. Guettier, B. Paule, E. Vibert, A. S. Cunha, D. Cherqui, D. Samuel, H. Bismuth, D. Castaing and R. Adam, J. Hepatol., 2015, 63, 83-92.

13 B. C. Odisio, F. Galastri, R. Avritscher, B. B. Afonso, V. Segatelli, G. E. Felga, P. R. Salvalaggio, J. Ensor, M. J. Wallace and F. Nasser, Cardiovasc. Intervent. Radiol., 2014, 37, 1018-1026.

14 W. H. Kang, S. Hwang, G. W. Song, Y. J. Lee, K. H. Kim, C. S. Ahn, D. B. Moon, D. H. Jung, G. C. Park and S. G. Lee, Liver Transplant., 2017, 23, 781-790.

15 J. DiNorcia, S. S. Florman, B. Haydel, P. Tabrizian, R. M. Ruiz, G. B. Klintmalm, S. Senguttuvan, D. D. Lee, C. B. Taner, E. C. Verna, K. J. Halazun, M. Hoteit,
M. H. Levine, W. C. Chapman, N. Vachharajani, F. Aucejo, M. H. Nguyen, M. L. Melcher, A. D. Tevar, A. Humar, C. Mobley, M. Ghobrial, T. L. Nydam, B. Amundsen, J. F. Markmann, J. Berumen, A. W. Hemming, A. N. Langnas, C. A. Carney, D. L. Sudan, J. C. Hong, J. Kim, M. A. Zimmerman, A. Rana, M. L. Kueht, C. M. Jones, T. M. Fishbein, D. Markovic, R. W. Busuttil and V. G. Agopian, Ann. Surg., 2020, 271, 616-624.

16 U. Asghar and T. Meyer, J. Hepatol., 2012, 56, 686-695.

17 T. Meyer, A. Kirkwood, M. Roughton, S. Beare, E. Tsochatzis, D. Yu, N. Davies, E. Williams, S. P. Pereira, D. Hochhauser, A. Mayer, R. Gillmore, J. O'Beirne, D. Patch and A. K. Burroughs, Br. J. Cancer, 2013, 108, 1252-1259.

18 K. T. Brown, R. K. Do, M. Gonen, A. M. Covey, G. I. Getrajdman, C. T. Sofocleous, W. R. Jarnagin, M. I. D’Angelica, P. J. Allen, J. P. Erinjeri, L. A. Brody, G. P. O'Neill, K. N. Johnson, A. R. Garcia, C. Beattie, B. Zhao, S. B. Solomon, L. H. Schwartz, R. DeMatteo and G. K. Abou-Alfa, J. Clin. Oncol., 2016, 34, 2046-2053.

19 N. R. Datta, S. G. Ordonez, U. S. Gaipl, M. M. Paulides, H. Crezee, J. Gellermann, D. Marder, E. Puric and S. Bodis, Cancer Treat. Rev., 2015, 41, 742-753.

20 M. A. Stuart, W. T. Huck, J. Genzer, M. Müller, C. Ober, M. Stamm, G. B. Sukhorukov, I. Szleifer, V. V. Tsukruk, M. Urban, F. Winnik, S. Zauscher, I. Luzinov and S. Minko, Nat. Mater., 2010, 9, 101-113.

21 A. Zhang, K. Jung, A. Li, J. Liu and C. Boyer, Prog. Polym. Sci., 2019, 99, 101164.

22 J. Seuring and S. Agarwal, Macromol. Rapid. Commun., 2012, 33, 1898-1920.

23 G. Huang, H. Li, S.-T. Feng, X. Li, G. Tong, J. Liu, C. Quan, Q. Jiang, C. Zhang and Z. Li, Macromol. Chem. Phys., 2015, 216, 1014-1023.

24 W. Li, L. Huang, X. Ying, Y. Jian, Y. Hong, F. Hu and Y. Du, Angew. Chem., Int. Ed., 2015, 54, 3126-3131.

25 C. Otsuka, Y. Wakahara, K. Okabe, J. Sakata, M. Okuyama, A. Hayashi, H. Tokuyama and S. Uchiyama, Macromolecules, 2019, 52, 7646-7660.

26 R. Lencioni and J. M. Llovet, Semin. Liver Dis., 2010, 30, 5260.

27 S. Tan, R. L. Sherman Jr. and W. T. Ford, Langmuir, 2004, 20, 7015-7020.

28 Z. Liu, Y. Tang, T. Kang, M. Rao, K. Li, Q. Wang, C. Quan, C. Zhang, Q. Jiang and H. Shen, Colloids Surf., B, 2015, 131, 39-46.

29 A. Poursaid, M. M. Jensen, E. Huo and H. Ghandehari, J. Controlled Release, 2016, 240, 414-433.

30 S. Tong, H. Zhu and G. Bao, Mater. Today, 2019, 31, 86-99.

31 M. Saeed, W. Ren and A. Wu, Biomater. Sci., 2018, 6, 708725.

32 M. Farokhi, F. Mottaghitalab, M. R. Saeb and S. Thomas, J. Controlled Release, 2019, 309, 203-219.

33 S. K. Calderwood and J. Gong, Trends Biochem. Sci., 2016, 41, 311-323.

34 J. Wang, J. Y. Chan, C. C. Fong, C. H. Tzang, K. P. Fung and M. Yang, Liver Int., 2009, 29, 1338-1347. 